\title{
Two Novel Fungal Symbionts Fusarium kuroshium sp. nov. and Graphium kuroshium sp. nov. of Kuroshio Shot Hole Borer (Euwallacea sp. nr. fornicatus) Cause Fusarium Dieback on Woody Host Species in California
}

Francis Na, Joseph D. Carrillo, Joey S. Mayorquin, Cedric Ndinga-Muniania, and Jason E. Stajich, Department of Plant Pathology and Microbiology, University of California, Riverside, 92521; Richard Stouthamer, Department of Entomology, University of California, Riverside, 92521; Yin-Tse Huang, Department of Plant Pathology, National Chung Hsing University, Taichung 402, Taiwan, ROC, and School of Forest Resources and Conservation, Institute of Food and Agricultural Sciences, University of Florida, Gainesville; Yu-Ting Lin and Chi-Yu Chen, Department of Plant Pathology, National Chung Hsing University, Taichung 402, Taiwan, ROC; and Akif Eskalen, ${ }^{\dagger}$ Department of Plant Pathology and Microbiology, University of California, Riverside, 92521

\begin{abstract}
Shot hole borer (SHB)-Fusarium dieback (FD) is a new pest-disease complex affecting numerous tree species in California and is vectored by two distinct, but related ambrosia beetles (Euwallacea sp. nr. fornicatus) called polyphagous shot hole borer (PSHB) and Kuroshio shot hole borer (KSHB). These pest-disease complexes cause branch dieback and tree mortality on numerous wildland and landscape tree species, as well as agricultural tree species, primarily avocado. The recent discovery of KSHB in California initiated an investigation of fungal symbionts associated with the KSHB vector. Ten isolates of Fusarium sp. and Graphium sp., respectively, were recovered from the mycangia of adult KSHB females captured in three different locations within San Diego County and compared with the known symbiotic fungi of PSHB. Multigene phylogenetic analyses of the internal transcribed spacer region (ITS), translation elongation

factor-1 alpha (TEF1- $\alpha$ ), and RNA polymerase II subunit (RPB1, RPB2) regions as well as morphological comparisons revealed that two novel fungal associates Fusarium kuroshium sp. nov. and Graphium kuroshium sp. nov. obtained from KSHB were related to, but distinct from the fungal symbionts $F$. euwallaceae and G. euwallaceae associated with PSHB in California. Pathogenicity tests on healthy, young avocado plants revealed $F$. kuroshium and G. kuroshium to be pathogenic. Lesion lengths from inoculation of $F$. kuroshium were found to be significantly shorter compared with those caused by $F$. euwallaceae, while no difference in symptom severity was detected between Graphium spp. associated with KSHB and PSHB. These findings highlight the pest disease complexes of KSHBFD and PSHB-FD as distinct, but collective threats adversely impacting woody hosts throughout California.
\end{abstract}

The Scolytinae is a subfamily (Alonso-Zarazaga and Lyal 2009) of bark and wood-boring weevils including more than 6,000 species that are of considerable economic importance in both temperate and tropical forests (Beaver et al. 2014; Smith and Hulcr 2015). Most of these insects typically attack declining or dead trees, but some are economically important species that attack apparently healthy trees in native and invaded areas, such as California. Ambrosia beetles are known to be associated with fungal and bacterial organisms (Beaver et al. 2014; Hulcr et al. 2012), with the fungal symbionts sometimes being phytopathogenic (Eskalen et al. 2013; Hulcr and Dunn 2011; Mendel et al. 2012; Ploetz et al. 2013). Shot hole borer (SHB)Fusarium dieback (FD) is an invasive pest-disease complex affecting numerous tree species in California. It was first reported on a host in 2012 (Eskalen et al. 2012) and has since been reported to attack over 300 tree species in wildland, landscape, and avocado growing areas (Eskalen et al. 2013; http://eskalenlab.ucr.edu/shotholeborerhosts.html). The extent of economic damage these pests have on native ecosystems in addition to agricultural settings in California is unknown, but the

${ }^{\dagger}$ Corresponding author: A. Eskalen; E-mail: akif.eskalen@ucr.edu

F. Na and J. D. Carrillo contributed equally to this work.

Funding: California Avocado Commission; University of California, Irvine; Orange County Parks; USDA National Institute of Food and Agriculture Hatch project CA-R-PPA-5061-H; USDA National Institute of Food and Agriculture Hatch project CA-R-PPA-5062-H; National Science Foundation MRI DBI-1429826 and NIH S10-OD016290.

*The $\boldsymbol{e}$-Xtra logo stands for "electronic extra" and indicates that two supplementary figures are published online.

Accepted for publication 19 December 2017.

C) 2018 The American Phytopathological Society estimated ecological and economic burdens in urban areas imposed by these invasive pests are significant. For example, since 2014, the PSHB-FD complex has infested $1 / 3$ of California sycamore (Platanus racemosa) in Orange County public parks and caused the removal of 1,262 trees, resulting in approximately US $\$ 4$ million in tree removal costs to Orange County parks (OC Parks 2017). Unfortunately, there are no viable management strategies to combat this pest disease complex besides removal of infested branches or trees to reduce beetle populations and hazards to people.

The negative impact ambrosia beetles and their mutualists are having on native and cultivated trees are not only a significant problem in California and other parts of the United States, but are also a significant problem in invaded and native areas throughout the world. For example, the Tea shot hole borer (Euwallacea fornicatus Eichoff, "TSHB") associated with Fusarium ambrosium is found in India and Sri Lanka (Danthanarayana 1968), where it is a serious pest of tea (Camellia sinensis). Invasive ambrosia beetles such as those belonging to the $E$. fornicatus species complex are native to southeast Asia (Beaver 1989; Hulcr and Stelinski 2017; Stouthamer et al. 2017) and have been previously described to form a unique mutualism with Fusarium spp. belonging to the Ambrosia Fusaria clade (AFC) (Kasson et al. 2013; O'Donnell et al. 2015). In addition to being associated with AFC members, ambrosia beetles from the Euwallacea genus have also been reported to be associated with other fungal genera including Graphium spp. (Beaver 1989; Freeman et al. 2016; Kasson et al. 2013; Kirisitis 2007; Lynch et al. 2016), Paracremonium spp. (Lynch et al. 2016), and also Raffaelea subfusca (Kasson et al. 2013) with Graphium spp. proposed to serve as a primary food source for developing larvae, reducing competition with adult females feeding on AFC fusaria (Freeman et al. 2016). Euwallacea spp. have been reported to invade multiple areas of the United States including California, Florida, Hawaii, and much of the mid-Atlantic and Southeastern U.S. (CABI 2015; Cognato et al. 2015; Eskalen et al. 2013; O'Donnell et al. 2015; Rabaglia et al. 2006; Short et al. 2017), as well as other parts of the world including Australia, Costa 
Rica, Guatemala, Israel, Panama, and South Africa (CABI 2015; Stouthamer et al. 2017). Invasions of more than a single Euwallacea species have occurred in California and Florida (Rabaglia et al. 2006; Stouthamer et al. 2017), with the former invaded by two genetically distinct, but morphologically indistinguishable Euwallacea spp. nr. fornicatus with independent evolutionary lineages (O'Donnell et al. 2015; Stouthamer et al. 2017), each associated with characteristic symbiotic fungi.

The two Euwallacea spp. invading California are now known as the polyphagous shot hole borer (Euwallacea sp. nr. fornicatus \#1., PSHB) (Eskalen et al. 2013; O'Donnell et al. 2015) and Kuroshio shot hole borer (Euwallacea sp. nr. fornicatus \#5, KSHB) (O'Donnell et al. 2015; Stouthamer et al. 2017). PSHB was initially reported on a backyard avocado in Los Angeles County in 2012 (Eskalen et al. 2012) and is known to be associated with three pathogenic fungal symbionts: F. euwallaceae S. Freeman, Z. Mendel, T. Aoki \& O'Donnell, Graphium euwallaceae M. Twizeyimana, S.C. Lynch \& A. Eskalen, and Paracremonium pembeum S.C. Lynch \& Eskalen. Since its initial discovery in 2012, PSHB has spread to several counties throughout southern California, including Orange, Riverside, San Bernardino, and Ventura (http://eskalenlab.ucr.edu/distribution.html). In 2013, new beetle infestations were detected on sycamore trees located in El Cajon in San Diego County. This beetle infestation was initially considered to be an extension of the existing infestation of PSHB in Los Angeles County. Molecular analysis of the fungal symbionts (Fusarium spp. and Graphium spp.) obtained from KSHB revealed significant differences from the symbionts recovered from PSHB in Los Angeles. The beetles collected from the San Diego infestation were also shown to be distinct from PSHB at the mitochondrial cytochrome oxidase I (COI) locus (Cooperband et al. 2016; Stouthamer et al. 2017). The beetles have since spread to adjacent counties and the two populations are currently sympatric in Orange and southern Los Angeles counties. Since there are currently two invasive SHB-FD complexes in California, it is important to characterize and distinguish the symbiotic fungi associated with the beetle vectors in order to elucidate any differences in pathogenicity, as well as describe morphological and genetic differences for diagnostic detection in affected areas.

In this study, we aimed to characterize the fungal symbionts of $\mathrm{KSHB}$ as well as explore the differences between the two groups of fungal symbionts associated with PSHB and KSHB. The primary objectives were to (i) identify morphological characteristics of Fusarium spp. and Graphium spp. associated with KSHB and resolve differences from other closely related species of the respective symbionts using multigene molecular phylogeny at informative loci, (ii) formally describe the novel fungal pathogens associated with KSHB as F. kuroshium and G. kuroshium, and (iii) determine the pathogenicity of $F$. kuroshium and G. kuroshium on healthy avocado plants and compare it to that of previously described $F$. euwallaceae (Freeman et al. 2013) and G. euwallaceae (Lynch et al. 2016) associated with PSHB.

\section{Materials and Methods}

Fungal isolate collection. Fungal isolates used in this study were obtained from the heads of beetles, similar to methods described by Lynch et al. (2016), and from wood recovered from KSHB galleries in their reproductive host trees, similar to methods described by Eskalen et al. (2013). A total of 40 beetles and symptomatic wood samples around the beetle galleries were collected from infested avocado (Persea americana) and sycamore (Platanus racemosa) in four different locations (Bonsall, El Cajon, Escondido, Fallbrook) in San Diego County. The samples were transferred to laboratory in a cooler (CDFA plant pest permit \#2887) and processed immediately. The beetles were surface sterilized by submerging in $70 \%$ ethanol and vortexed for $20 \mathrm{~s}$, rinsed with sterile deionized water, and allowed to dry on sterile filter paper. Beetle heads were separated from the thoracic and abdominal segments under a dissection microscope, then the segments were macerated in $1.5 \mathrm{ml}$ microcentrifuge tubes with sterile plastic pestles. The macerated heads were suspended in $1 \mathrm{ml}$ of sterile water and $50 \mu \mathrm{l}$ of the suspensions were pipetted onto Petri plates containing potato dextrose agar (PDA; BD Difco, Sparks, MD) amended with tetracycline hydrochloride
$(0.01 \%)$ and spread using sterile glass rods. Plates were incubated for 5 days at $25^{\circ} \mathrm{C}$ and fungal colonies were counted and unique morphologies were subcultured for further identification. Five replication plates per beetle head suspension were used to quantify relative fungal content in their mycangia similar to methods described by Lynch et al. (2016). Beetle gallery wall samples were obtained by carving out small pieces of galleries from infested sycamore and avocado wood and sampling of the gallery surfaces was performed by scraping the gallery wall with a sterile wooden toothpick (Kajimura and Hijii 1992). Wooden toothpicks containing the scrapings were rinsed with $1 \mathrm{ml}$ of sterile water in a microcentrifuge tube, and $50 \mu \mathrm{l}$ of the wash were pipetted onto plates containing PDA amended with tetracycline hydrochloride $(0.01 \%)$ and spread using sterile glass rods. Plates were incubated at $25^{\circ} \mathrm{C}$ for 5 days, and individual, singlespore fungal colonies were isolated from the plates.

Fungal DNA extraction, PCR, and phylogenetic analysis. Genomic DNA of the fungal isolates obtained from KSHB beetles and gallery samples was extracted using a modified protocol by Cenis (1992). Briefly, $50 \mu \mathrm{g}$ of fungal mycelia was harvested from 7-dayold Fusarium spp. or 14-day-old Graphium spp. culture plates and added to microcentrifuge tubes containing glass beads and $500 \mu \mathrm{lex}-$ traction buffer ( $200 \mathrm{mM}$ Tris HCL, $250 \mathrm{mM} \mathrm{NaCl}, 25 \mathrm{mM}$ EDTA, $2 \%$ sodium dodecyl sulfate in $250 \mathrm{ml} \mathrm{H}_{2} \mathrm{O}$ ), then the mixture was processed in a tissue homogenizer (MP Biomedicals, Irvine, CA) to lyse. Sodium acetate $(150 \mu \mathrm{l})$ was then added to each microcentrifuge tube, and they were placed in a $-20^{\circ} \mathrm{C}$ freezer for $10 \mathrm{~min}$. The microcentrifuge tubes were centrifuged at 14,000 relative centrifugal force (RCF), and then $300 \mu \mathrm{l}$ of the supernatants were mixed with an equal volume of isopropanol prior to storing at $0^{\circ} \mathrm{C}$ for $10 \mathrm{~min}$. The precipitated DNA was pelleted by centrifugation at 14,000 RCF, washed with $70 \%$ ethanol, air dried, then suspended in TE buffer.

PCR amplification of the internal transcribed spacer (ITS)1-5.8SITS2 and translation elongation factor $1-\alpha($ TEF1- $\alpha)$ was done using ITS4/ITS5 (White et al. 1990) for all species tested, EF1/EF2 for Fusarium spp. only (O'Donnell et al. 1998), and EF1F/EF2R for Graphium spp. only (Jacobs et al. 2004). In addition, PCR amplification of RNA polymerase subunit I (RPB1) and RNA polymerase subunit II (RPB2) loci was performed for Fusarium spp. only using primers F5/R8 (RPB1-1) (O'Donnell et al. 2010), F7/G2R (RPB12) (O'Donnell et al. 2010), 5F2/7CR (RPB2-1) (O'Donnell et al. 2007), and 7CF/11AR (RPB2-2) (O'Donnell et al. 2007). It should be noted that large subunit (LSU) domains D1 and D2 of the LSU rDNA partition were previously found to be least informative (Kasson et al. 2013; O'Donnell et al. 2015), and therefore were not included in this study. Each PCR reaction mixture consisted of $12.5 \mu \mathrm{l}$ GoTaq DNA polymerase (Promega, Madison, WI), $9.3 \mu \mathrm{l}$ sterile DNase-free water, $0.6 \mu \mathrm{l}$ of $10 \mu \mathrm{M}$ forward primer, $0.6 \mu \mathrm{l}$ of $10 \mu \mathrm{M}$ reverse primer PCR, and $2 \mu \mathrm{l}$ of genomic DNA template, for a total of $25 \mu \mathrm{l}$ reaction mixture. PCR was performed for each primer set using published cycling parameters (Jacobs et al. 2004; O'Donnell et al. 1998, 2007; White et al. 1990). Amplified products were separated by gel electrophoresis in $1 \%$ agarose gel with $0.5 \times$ Tris-boric acid-EDTA buffer, stained with SYBR Green (Invitrogen, Carlsbad, CA), and viewed under UV light. Products were purified using ExoSAP-IT (Affymetrix, Santa Clara, CA), then sequenced in both directions at the Institute for Integrative Genome Biology, University of California Riverside with corresponding primers used for PCR. Raw sequences were assembled in Sequencher 4.6 (Gene Codes Corp., Ann Arbor, MI).

Multigene phylogenetic analysis was conducted to determine the genetic relatedness of Fusarium sp. and Graphium sp. isolates obtained from sampled locations where KSHB is present. These isolates were also compared with other members of their respective genera. Phylogenetic analysis of Fusarium spp. was conducted using concatenated DNA sequences at ITS, TEF1- $\alpha$, RPB1, and RPB2 gene regions from 10 isolates of Fusarium spp., DNA of 10 isolates of Fusarium spp. native to Taiwan (provided by Dr. Chi-Yu Chen) (Table 1), along with DNA sequences obtained from GenBank (Table 2) from 26 isolates previously used in AFC phylogenetic analysis from Kasson et al. (2013) and O'Donnell et al. (2015). Phylogenetic analysis of 
Graphium spp. was conducted using concatenated DNA sequences of ITS and TEF1- $\alpha$ gene regions from 10 isolates of Graphium spp. (Table 1) as well as 36 isolates obtained from GenBank (Table 2) used in previous analysis done by Lynch et al. (2016). All respective sequences from Fusarium spp. and Graphium spp. analysis used were aligned using Clustal X (Thompson et al. 1997) and concatenated after alignment. A partition file was created to indicate the range of each gene in the concatenated alignment and allow for different substitution models. The multigene phylogenies were constructed with maximum likelihood (ML) methods RAxML version 8.2.8 (Stamatakis 2014) and IQ-TREE (Nguyen et al. 2015). PartitionFinder was used to determine that the best partitioning scheme was EF, ITS, and RPB1+RPB2. RAxML was run with the rapid bootstrap approach, generating 1,000 bootstrap replicates, followed by a thorough ML search. IQ-TREE was run with 1,000 standard bootstrap analyses to generate final tree run. The ModelFinder option identified the best substitution model for each of the three defined partitions (-m MFP -nt AUTO -spp partitions.txt -b 1000) for Fusarium spp. and for each of the two defined partitions (-bb 1000 -alrt 1000 -m TESTNEW -q partitions.txt) for Graphium spp. tested.

Morphological characterization. Morphological characteristics of Fusarium spp. and Graphium spp. were observed using a compound microscope (Leica DMLB, Wetzlar, Germany) with a SPOT camera attachment (Diagnostic Instruments, Sterling Heights, MI) using 10 isolates of each fungal species. Fusarium spp. cultures were grown on PDA and synthetic low-nutrient agar (SNA; Nirenberg 1976) in complete darkness, under near UV light (F40T12/BLB 40W, Philips, Amsterdam, Netherlands), and under constant light (F40T12/CW 40W, Philips, Amsterdam, Netherlands), at ambient room temperature (23 to $25^{\circ} \mathrm{C}$ ), and examined at 14 days and 1 month. Graphium spp. cultures were grown on PDA and oat meal agar (OMA) (Gams et al. 1998) under constant light (F40T12/CW 40W, Philips, Amsterdam, Netherlands) at ambient room temperature and examined at 14 days. Cultures of Fusarium spp. on PDA in 6-cm Petri dishes at $25^{\circ} \mathrm{C}$ in darkness were used to characterize colony color and morphology. Fourteenday-old cultures of Fusarium spp. were used to evaluate microscopic characters on SNA and PDA similar to Aoki et al. (2005) and Freeman et al. (2013). The Munsell (1976) color palette was used as the color standard. Cultures of Graphium spp. were grown on PDA in 6-cm Petri dishes at $25^{\circ} \mathrm{C}$ to evaluate colony color, morphology, and microscopic characters. The lengths and widths of the Fusarium spp. and Graphium spp. conidia were measured from cultures using SPOT imaging software (Diagnostic Instruments, Sterling Heights, MI).

Growth rates of three isolates of each Fusarium spp. and Graphium spp., respectively, were obtained by measuring the fungal colony diameters of cultures incubated at various temperatures between 5 and $40^{\circ} \mathrm{C}\left(5^{\circ} \mathrm{C}\right.$ increments $)$ in replicates of three. Plugs of agar containing the fungi were obtained from the edges of 1-week-old cultures and placed on Petri plates containing PDA. Diameters of the Fusarium spp. colonies were measured every day for 9 days, and Graphium spp. were measured every 2 days for 14 days. This experiment was repeated once.

Pathogenicity assay. Pathogenicity assays were conducted under greenhouse conditions, in which 2-year-old avocado cv. Zutano seedlings were each inoculated with F. euwallaceae, Fusarium spp., G. euwallaceae, or Graphium spp. using similar methods described by Twizeyimana et al. (2013). Two isolates from each fungal species were used in the pathogenicity assay, and each treatment was assigned by complete randomized design with 10 replicates across two trials. Ten avocado plants were used per fungal species and control in each

Table 1. Representative isolates of Fusarium spp. and Graphium spp. from Euwallacea spp. and various hosts obtained in this study

\begin{tabular}{|c|c|c|c|c|c|c|c|}
\hline \multirow[b]{2}{*}{ Isolate } & \multirow[b]{2}{*}{ Species } & \multirow[b]{2}{*}{ Host } & \multirow[b]{2}{*}{ Location $^{\mathbf{a}}$} & \multicolumn{4}{|c|}{ GenBank numbers ${ }^{b}$} \\
\hline & & & & ITS & EF1- $\alpha$ & RPB1 & RPB2 \\
\hline$\overline{\mathrm{UCR} 3641^{\mathrm{cd}}}$ & Fusarium sp. & Platanus racemosa & El Cajon, CA & KX262196 & KX262216 & KX262236 & KX262256 \\
\hline UCR3644d & Fusarium sp. & $P$. racemosa & El Cajon, CA & KX262197 & KX262217 & KX262237 & KX262257 \\
\hline UCR3651 & Fusarium sp. & Euwallacea sp. & Fallbrook, CA & KX262198 & KX262218 & KX262238 & KX262258 \\
\hline UCR3653 & Fusarium sp. & Persea americana & Bonsall, CA & KX262200 & KX262220 & KX262240 & KX262260 \\
\hline UCR3654 & Fusarium sp. & P. americana & Bonsall, CA & KX262201 & KX262221 & KX262241 & KX262261 \\
\hline UCR3657 & Fusarium sp. & Euwallacea sp. & Bonsall, CA & KX262202 & KX262222 & KX262242 & KX 262262 \\
\hline UCR3659 & Fusarium sp. & Euwallacea sp. & Bonsall, CA & KX262203 & KX262223 & KX262243 & KX262263 \\
\hline UCR3660 & Fusarium sp. & Euwallacea sp. & Bonsall, CA & KX262204 & KX262224 & KX262244 & KX262264 \\
\hline UCR3661 & Fusarium sp. & P. americana & Escondido, CA & KX262205 & KX262225 & KX262245 & KX262265 \\
\hline UCR3662 & Fusarium sp. & P. americana & Escondido, CA & KX262199 & KX262219 & KX262239 & KX262259 \\
\hline $\mathrm{UCR} 4672^{\mathrm{e}}$ & Fusarium sp. & Euwallacea sp. & Taichung, Taiwan & KX262206 & KX262226 & KX262246 & KX262266 \\
\hline UCR4673 & Fusarium sp. & Euwallacea sp. & Taichung, Taiwan & KX262207 & KX262227 & KX262247 & KX262267 \\
\hline $\mathrm{UCR} 4674^{\mathrm{e}}$ & Fusarium sp. & Euwallacea sp. & Taichung, Taiwan & KX262208 & KX262228 & KX262248 & KX262268 \\
\hline $\mathrm{UCR} 4675^{\mathrm{e}}$ & Fusarium sp. & Euwallacea sp. & Taichung, Taiwan & KX262209 & KX262229 & KX262249 & KX262269 \\
\hline $\mathrm{UCR} 4676^{\mathrm{e}}$ & Fusarium sp. & Euwallacea sp. & Taichung, Taiwan & KX262210 & KX262230 & KX262250 & KX262270 \\
\hline UCR4677e & Fusarium sp. & Euwallacea sp. & Taichung, Taiwan & KX262211 & KX262231 & KX262251 & KX262271 \\
\hline UCR $4678^{\mathrm{e}}$ & Fusarium sp. & Euwallacea sp. & Taichung, Taiwan & KX262212 & KX262232 & KX262252 & KX262272 \\
\hline UCR4679e & Fusarium sp. & Euwallacea sp. & Taichung, Taiwan & KX262213 & KX262233 & KX262253 & KX262273 \\
\hline $\mathrm{UCR} 4680^{\mathrm{e}}$ & Fusarium sp. & Euwallacea sp. & Taichung, Taiwan & KX262214 & KX262234 & KX262254 & KX262274 \\
\hline $\mathrm{UCR} 4681^{\mathrm{e}}$ & Fusarium sp. & Euwallacea sp. & Taichung, Taiwan & KX262215 & KX262235 & KX262255 & KX262275 \\
\hline UCR4593cd & Graphium sp. & P. americana & Fallbrook, CA & KX262276 & KX262286 & $\ldots$ & $\ldots$ \\
\hline UCR4594 ${ }^{\mathrm{d}}$ & Graphium sp. & P. americana & Fallbrook, CA & KX262277 & KX262287 & $\ldots$ & $\ldots$ \\
\hline UCR4606 & Graphium sp. & P. americana & Bonsall, CA & KX262278 & KX262288 & $\ldots$ & $\ldots$ \\
\hline UCR4607 & Graphium sp. & P. americana & Bonsall, CA & KX262279 & KX262289 & $\ldots$ & $\ldots$ \\
\hline UCR4608 & Graphium sp. & Euwallacea sp. & Bonsall, CA & KX262280 & KX262290 & $\ldots$ & $\ldots$ \\
\hline UCR4609 & Graphium sp. & Euwallacea sp. & Bonsall, CA & KX262281 & KX262291 & $\ldots$ & $\ldots$ \\
\hline UCR4616 & Graphium sp. & P. americana & Escondido, CA & KX262282 & KX262292 & $\ldots$ & $\ldots$ \\
\hline UCR4617 & Graphium sp. & P. americana & Escondido, CA & KX262283 & KX262293 & $\ldots$ & $\ldots$ \\
\hline UCR4618 & Graphium sp. & Euwallacea sp. & Escondido, CA & KX262284 & KX262294 & $\ldots$ & $\ldots$ \\
\hline UCR4622 & Graphium sp. & Euwallacea sp. & Escondido, CA & KX262285 & KX262295 & $\ldots$ & $\ldots$ \\
\hline
\end{tabular}

a Taiwanese fungal isolates collected and sequenced by C. Y. Chen.

b ITS: internal transcribed spacer regions; EF1- $\alpha$ : translation elongation factor 1- $\alpha$; RPB: RNA II polymerase subunit.

${ }^{c}$ Denotes type-specimen.

${ }^{\mathrm{d}}$ Isolates used in pathogenicity test.

e DNA only. 
Table 2. Descriptions and sequences of fungi obtained from GenBank used in the phylogenetic analysis

\begin{tabular}{|c|c|c|c|c|c|c|c|}
\hline \multirow[b]{2}{*}{ Isolate } & \multirow[b]{2}{*}{ Species } & \multirow[b]{2}{*}{ Host } & \multirow[b]{2}{*}{ Origin } & \multicolumn{4}{|c|}{ GenBank numbers ${ }^{a}$} \\
\hline & & & & ITS & EF1- $\alpha$ & RPB1 & $\mathbf{R P B 2}^{\mathbf{b}}$ \\
\hline NRRL20438 & Fusarium ambrosium [AF-1] & Euwallacea fornicatus & India & AF178397 & AF178332 & JX171470 & JX171584 \\
\hline NRRL22231 & Fusarium sp. [AF-5] & Hevea brasiliensis & Malaysia & KC691570 & KC691542 & KC691600 & КС691631, КС691660 \\
\hline NRRL22346 & F. ambrosium [AF-1] & E. fornicatus & India & EU329669 & FJ240350 & KC691587 & EU329503 \\
\hline NRRL22468 & F. neocosmosporiellum & Arachis hypogaea & Guinea & DQ094318 & AF178349 & KC691616 & EU329512 \\
\hline NRRL22643 & Fusarium sp. [AF-9] & Xyleborus ferrigineus & Costa Rica & KC691583 & DQ247628 & KC691613 & КС691644, КС691673 \\
\hline NRRL32434 & F. lichenicola & Homo sapiens & Germany & DQ094444 & DQ246977 & HМ347156 & EF470161 \\
\hline NRRL43467 & F. neocosmosporiellum & Homo sapiens & LA (U.S.A.) & EF453092 & EF452940 & HM347178 & EF469979 \\
\hline NRRL46518 & Fusarium sp. [AF-5] & H. brasiliensis & Malaysia & KC691571 & KC691543 & KC691601 & КС691632, КС691661 \\
\hline NRRL54722 & Fusarium sp. [AF-2] & Euwallacea sp. & Israel & JQ038014 & JQ038007 & JQ038021 & JQ038028 \\
\hline NRRL54723 & Fusarium sp. [AF-2] & Euwallacea sp. & Israel & JQ038015 & JQ038008 & JQ038022 & JQ038029 \\
\hline NRRL54724 & Fusarium sp. [AF-2] & Euwallacea sp. & Israel & JQ038016 & JQ038009 & JQ038023 & JQ038030 \\
\hline NRRL54725 & Fusarium sp. [AF-2] & Euwallacea sp. & Israel & JQ038017 & JQ038010 & JQ038024 & JQ038031 \\
\hline NRRL54726 & Fusarium sp. [AF-2] & Euwallacea sp. & Israel & JQ038018 & JQ038011 & JQ038025 & JQ038032 \\
\hline NRRL54727 & Fusarium sp. [AF-2] & Euwallacea sp. & Israel & JQ038019 & JQ038012 & JQ038026 & JQ038033 \\
\hline NRRL62578 & Fusarium sp. [AF-4] & E. validus & PA (U.S.A.) & KC691565 & KC691537 & KC691595 & КС691626, КС691655 \\
\hline NRRL62579 & Fusarium sp. [AF-4] & E. validus & PA (U.S.A.) & KC691566 & KC691538 & KC691596 & КС691627, КС691656 \\
\hline NRRL62584 & Fusarium sp. [AF-8] & Euwallacea sp. & FL (U.S.A.) & KC691582 & KC691554 & KC691612 & КС691643, КС691672 \\
\hline NRRL62585 & Fusarium sp. [AF-8] & Euwallacea sp. & FL (U.S.A.) & KC691577 & KC691549 & KC691607 & КС691638, КС691667 \\
\hline NRRL62590 & Fusarium sp. [AF-6] & Euwallacea sp. & FL (U.S.A.) & KC691574 & KC691546 & KC691604 & КС691635, КС691664 \\
\hline NRRL62591 & Fusarium sp. [AF-6] & Euwallacea sp. & FL (U.S.A.) & KC691573 & KC691545 & KC691603 & КС691634, КС691663 \\
\hline NRRL62606 & Fusarium sp. [AF-3] & Euwallacea sp. & FL (U.S.A.) & KC691561 & KC691533 & KC691591 & КС691622, КС691651 \\
\hline NRRL62610 & Fusarium sp. [AF-7] & Euwallacea sp. & Australia & KC691575 & KC691547 & KC691605 & КС691636, КС691665 \\
\hline NRRL62611 & Fusarium sp. [AF-7] & Euwallacea sp. & Australia & KC691576 & KC691548 & KC691606 & КС691637, КС691666 \\
\hline NRRL62629 & Fusarium sp. [AF-3] & E. interjectus & FL (U.S.A.) & KC691564 & KC691536 & KC691594 & КС691625, КС691654 \\
\hline NRRL62941 & Fusarium sp. [AF-10] & unknown & Singapore & KM406633 & KM406626 & KM406640 & KM406647 \\
\hline NRRL62942 & F. ambrosium & Camellia sinensis & Sri Lanka & KM406631 & KM406624 & KM406638 & KM406645 \\
\hline NRRL62944 & Fusarium sp. [AF-11] & C. sinensis & Sri Lanka & KM406634 & KM406627 & KM406641 & KM406648 \\
\hline NRRL66088 & Fusarium sp. & Delonix regia & FL (U.S.A.) & KM406632 & KM406632 & KM406639 & KM406646 \\
\hline UCR2974 & Graphium euwallaceae & Ricinus communis & CA (U.S.A.) & KF540218 & KF534799 & $\ldots$ & $\ldots$ \\
\hline UCR2975 & G. euwallaceae & Acer negundo & CA (U.S.A.) & KF540219 & KF534800 & $\ldots$ & $\ldots$ \\
\hline UCR2976 & G. euwallaceae & R. communis & CA (U.S.A.) & KF540220 & KF534801 & $\ldots$ & $\ldots$ \\
\hline UCR2977 & G. euwallaceae & Acacia floribunda & CA (U.S.A.) & KF540221 & KF534802 & $\ldots$ & $\ldots$ \\
\hline UCR2978 & G. euwallaceae & Erythrina atitlanensis & CA (U.S.A.) & KF540222 & KF534803 & $\ldots$ & $\ldots$ \\
\hline UCR2979 & G. euwallaceae & Quercus agrifolia & CA (U.S.A.) & KF540223 & KF534804 & $\ldots$ & $\ldots$ \\
\hline UCR2980 & G. euwallaceae & Persea americana & CA (U.S.A.) & KF540224 & KF534805 & $\ldots$ & $\ldots$ \\
\hline UCR2981 & G. euwallaceae & P. americana & CA (U.S.A.) & KF540225 & KF534806 & $\ldots$ & $\ldots$ \\
\hline UCR2308 & G. euwallaceae & Acacia auriculiformis & Vietnam & KM592371 & KM592363 & $\ldots$ & $\ldots$ \\
\hline UCR2159 & Graphium sp. I & Ailanthus altissima & PA (U.S.A.) & KJ131228 & KJ131238 & $\ldots$ & $\ldots$ \\
\hline UCR2160 & Graphium sp. I & A. altissima & PA (U.S.A.) & KJ131229 & KJ131239 & $\ldots$ & $\ldots$ \\
\hline UCR2162 & Graphium sp. I & A. altissima & PA (U.S.A.) & KJ131231 & KJ131241 & $\ldots$ & $\ldots$ \\
\hline UCR2163 & Graphium sp. I & A. altissima & PA (U.S.A.) & KJ131232 & KJ131242 & $\ldots$ & $\ldots$ \\
\hline UCR2164 & Graphium sp. I & A. altissima & PA (U.S.A.) & KJ131233 & KJ131243 & $\ldots$ & $\ldots$ \\
\hline UCR2165 & Graphium sp. I & A. altissima & PA (U.S.A.) & KJ131234 & KJ131244 & $\ldots$ & $\ldots$ \\
\hline UCR2166 & Graphium sp. I & A. altissima & PA (U.S.A.) & KJ131235 & KJ131245 & $\ldots$ & $\ldots$ \\
\hline UCR2132 & Graphium sp. II & Durio sp. & Thailand & KM592367 & KM363259 & $\ldots$ & $\ldots$ \\
\hline UCR2137 & Graphium sp. II & Durio sp. & Thailand & KJ131236 & KJ131246 & $\ldots$ & $\ldots$ \\
\hline UCR2140 & Graphium sp. II & Durio sp. & Thailand & KJ131237 & KJ131247 & $\ldots$ & $\ldots$ \\
\hline UCR2289 & Graphium sp. III & A. auriculiformis & Vietnam & KM592368 & KM592360 & $\ldots$ & $\ldots$ \\
\hline UCR2291 & Graphium sp. III & A. auriculiformis & Vietnam & KM592369 & KM592361 & $\ldots$ & $\ldots$ \\
\hline UCR2300 & G. carbonarium & A. auriculiformis & Vietnam & KM592370 & KM592362 & $\ldots$ & $\ldots$ \\
\hline UCR2325 & G. carbonarium & $R$. communis & Vietnam & KM592372 & KM592364 & $\ldots$ & $\ldots$ \\
\hline UCR2329 & G. carbonarium & R. communis & Vietnam & KM592373 & KM592365 & $\ldots$ & $\ldots$ \\
\hline CMW12418 & G. carbonarium & Salix babylonica & China & FJ434980 & HM630602 & $\ldots$ & $\ldots$ \\
\hline CMW12420 & G. carbonarium & S. babylonica & China Salomon & FJ434989 & HM630603 & $\ldots$ & $\ldots$ \\
\hline JCM 9300 & G. basitruncatum & Forest soil & Islands & $\mathrm{AB} 038427$ & KJ131248 & $\ldots$ & $\ldots$ \\
\hline CMW30626 & G. fabiforme & Adansonia rubrostipa & Madagascar & GQ200616 & HM630592 & $\ldots$ & $\ldots$ \\
\hline CMW30627 & G. fabiforme & A. rubrostipa & Madagascar & GQ200617 & HM630593 & $\ldots$ & $\ldots$ \\
\hline CMW5605 & G. fimbriisporum & Picea abies & France & AY148177 & HM630590 & $\ldots$ & $\ldots$ \\
\hline CMW5606 & G. fimbriisporum & P. abies & Austria & AY148180 & HM630591 & $\ldots$ & $\ldots$ \\
\hline CMW5601 & G. laricis & Larix decidua & Austria & AY148183 & HM630588 & $\ldots$ & $\ldots$ \\
\hline CMW5603 & G. laricis & L. decidua & Austria & AY148182 & HM630589 & $\ldots$ & $\ldots$ \\
\hline CMW5292 & G. penicillioides & Populus nigra & Czech Republic & HQ335310 & HM630600 & $\ldots$ & $\ldots$ \\
\hline CMW5295 & G. penicillioides & P. nigra & Czech Republic & HQ335311 & HM630601 & $\ldots$ & $\ldots$ \\
\hline CMW503c & G. pseudormiticum & Pinus sp. & South Africa & AY148186 & HM630586 & $\ldots$ & $\ldots$ \\
\hline CMW12285 & G. pseudormiticum & Tsuga dumosa & China & HM630608 & HM630587 & $\ldots$ & $\ldots$ \\
\hline
\end{tabular}

a ITS = internal transcribed spacer region; EF1- $\alpha=$ translation elongation factor 1- $\alpha$; RPB = DNA-directed RNA polymerase II subunit.

b Two accession numbers correspond to unjoined RPB2-1 and RPB2-2 sequences.

${ }^{\mathrm{c}}$ Denotes type-specimens. 
trial. The fungal cultures used during this experiment were grown on PDA for 7 days (F. euwallaceae, Fusarium sp.) or 14 days (G. euwallaceae, Graphium sp.) at $25^{\circ} \mathrm{C}$, and agar plugs $5 \mathrm{~mm}$ in diameter were cut from the cultures with a sterile cork borer. Upon inoculation, the 5-mm-diameter cork borer was flame-sterilized and used to create wounds in the avocado stems by boring into the xylem to allow pathogens to colonize the wood tissue. The wounds were filled with the agar plugs containing the fungal mycelium side down, then wrapped with Parafilm coated with petroleum jelly. Control plants were wounded in the same manner with a cork borer and sterile agar plugs were applied to the wounds. The inoculated plants were grown for 30 days in the greenhouse prior to destructive sampling.

The wood tissues of the inoculated plants were examined for visible lesions (necrotic tissue) originating from points of fungal inoculation and lesion lengths were measured from the edges of visible discoloration. Wood tissue from the xylem was collected from the margins of lesions, embedded on PDA plates amended with tetracycline hydrochloride $(0.01 \%)$, and incubated at $25^{\circ} \mathrm{C}$ for 5 days to recover the fungi from the inoculated plants. Presence of Fusarium or Graphium in each xylem sample was verified by culturing and subsequent identification sequencing the TEF1- $\alpha$ gene to fulfill Koch's postulates.

Statistical analyses. All statistical analyses were performed using $\mathrm{R}$ (version 3.2.3). Analysis of microscopic characters were performed by analysis of variance (ANOVA) using the CAR package (Fox et al. 2016) and means, standard deviation, standard error, max, and min values were calculated implementing the PLYR package (Wickham 2011). Xylem lesion lengths were analyzed by using regression in the LME4 package (Bates et al. 2015) to model the response variable of lesion length by independent factors: fungal species ( $F$. euwallaceae, Fusarium sp., G. euwallaceae, and Graphium sp.) and trial. Data were transformed via $\log _{10}$ data transformation but the assumption of normality and homogeneity of variance were both violated. Therefore, a post hoc Tukey's bootstrap test was performed at $\alpha=0.05$ with specified contrast to control groups. All plots were made using Sigma Plot (version 11; Systat Software Inc., Point Richmond, CA).

\section{Results}

Phylogenetic analysis. Sequences of $F$. neocosmosporiellum (NRRL 22468, NRRL 43467) and $F$. lichenicola (NRRL 32434) were used as outgroups for rooting the Fusarium trees based on prior analyses of AFC members (Kasson et al. 2013; O'Donnell et al. 2015).
Multilocus phylogenetic analysis performed on four loci (ITS, TEF1$\alpha$, RPB1, and RPB2) from Fusarium isolates tested in this study indicate Fusarium sp. (AF-12) recovered from KSHB as a distinct phylogenetic species from $F$. euwallaceae (AF-2) recovered from PSHB and other closely related Fusarium spp. (minimum $91 \%$ bootstrap support) (Fig. 1, Supplementary Fig. S1). Statistical confidence in the phylogenetic clades was assessed by comparing bootstrap support across multiple analysis methods (maximum likelihood, maximum parsimony [data not shown], neighbor-joining [data not shown]). The recovered tree topologies found strong support for reciprocal monophyly of the $F$. euwallaceae (AF-2) strains (NRRL 54722-54726) and Fusarium sp. (AF-12) strains (UCR 3641, 3644, 3651-3654, 3657-3661) analyzed. All Fusarium sp. (AF-12) strains and Fusarium spp. strains from Taiwan (UCR 4672-4681) reside in AFC major clade B (Kasson et al. 2013; O'Donnell et al. 2015) containing AF-[1-7;1012] strains; no isolates examined in this study were found to be members of AFC clade A containing AF-[8-9] strains. Strains recovered from Euwallacea spp. originating from Taiwan are paraphyletic, with the AF-[13-15] strains showing closer relation to $F$. euwallaceae (AF-2), Fusarium sp. (AF-12), and Fusarium spp. (AF-[3,4]) compared with AF-16 strains, which are sister lineages to this resolved clade.

Four phylogenetic species (AF-[13-16]) recovered from beetles in Taiwan can be identified from our analysis and were found to be largely divided into four groups. Fusarium sp. AF-13 (UCR 4674, 4675) is sister to Fusarium sp. (AF-12). Fusarium sp. AF-14 Taiwan (UCR 4672, 4681) is part of an unresolved clade and equidistant to the closest relatives $F$. euwallaceae (AF-2), Fusarium spp. (AF-12), and Fusarium sp. AF-3 FL (U.S.A.) (NRRL 62606, 62629). Fusarium sp. AF-15 Taiwan (UCR4679) is sister to Fusarium sp. AF-4 Pennsylvania (U.S.A.) (NRRL 62578, 62579). Fusarium sp. AF-16 Taiwan (UCR4680, 4673, 4678, 4676, 4677) forms a distinct monophyletic clade that is sister to the clade containing the prior described lineages of AF-[2-4;12-15].

Sequences from previous phylogenetic analysis of Graphium spp. (Lynch et al. 2016) were used to build an unrooted phylogenetic tree (Fig. 2, Supplementary Fig. S2). Graphium sp. recovered from KSHB in California were found to be genetically distinct from G. euwallaceae recovered from PSHB California through multilocus phylogenetic analysis using two informative loci, ITS and TEF1- $\alpha$. The phylogenetic analysis resolved all Graphium sp. isolates associated with

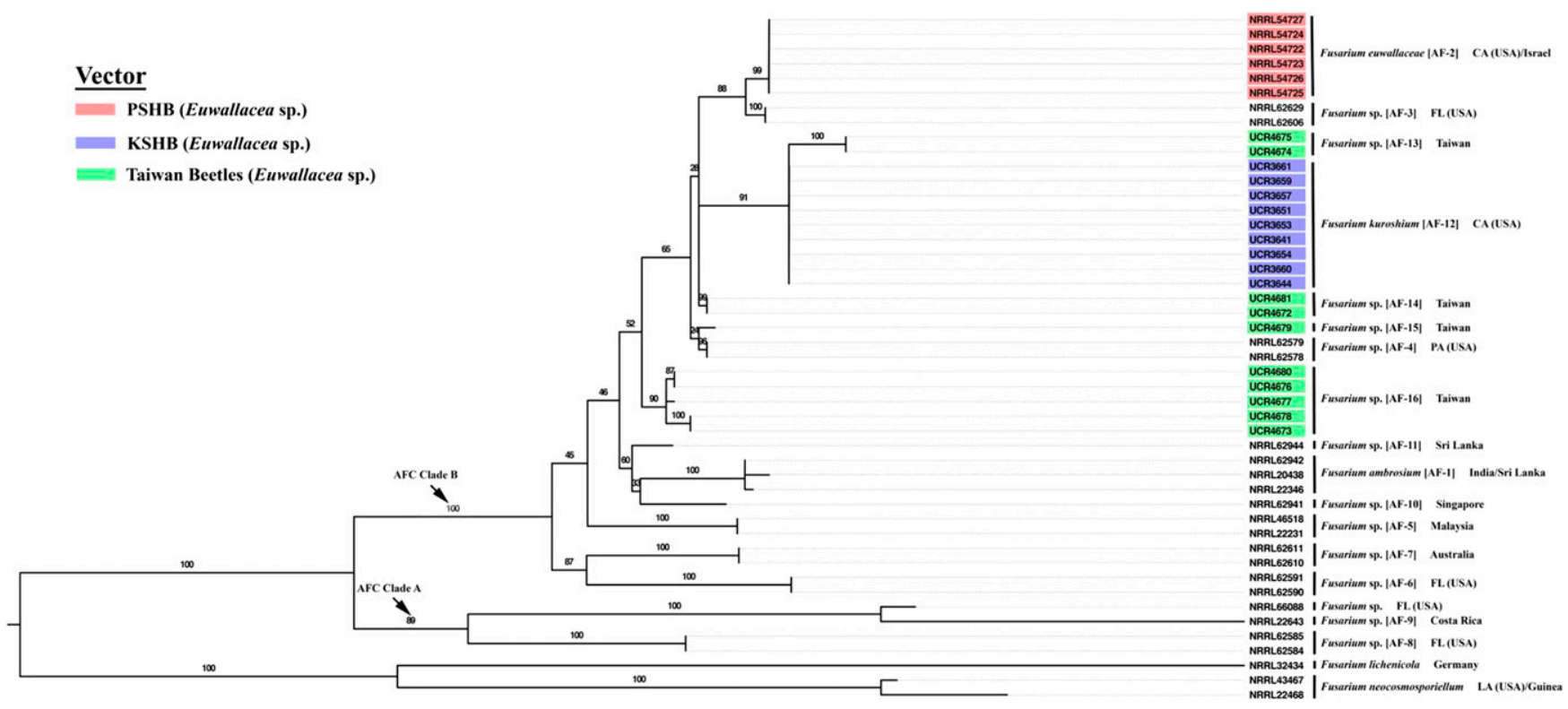

Fig. 1. Multilocus phylogenetic analysis of Ambrosia Fusaria clade (AFC) fusaria conducted with four genes: ribosomal internal transcribed spacer (ITS), elongation factor 1- $\alpha$ (EF1- $\alpha$ ), DNA-directed RNA polymerase II largest subunit (RPB1), and DNA-directed RNA polymerase II second largest subunit (RPB2). Diagram was constructed using IQ-TREE maximum likelihood method bootstrapped with 1,000 replications. The highlighted boxes indicate the beetle vector the fungi were recovered from. 
KSHB in a monophyletic clade ( $83 \%$ bootstrap support) that was distinct from a well-supported clade of $G$. euwallaceae isolates $(94 \%$ bootstrap support). Statistical confidence in the phylogenetic clades was assessed by comparing bootstrap support across multiple analysis methods (maximum likelihood, maximum parsimony [data not shown], and neighbor-joining [data not shown]). The recovered tree topologies found strong support for reciprocal monophyly of the G. euwallaceae strains (UCR 2308, 2974-2981) and Graphium sp. strains (UCR 4593, 4594, 4606-4609, 4616-4618, 4622) analyzed. Isolates associated with wood-boring beetles form a well-supported clade that contained G. euwallaceae, G. carbonarium, Graphium spp. (I-III) (Lynch et al. 2016), and Graphium sp. (KSHB). G. basitruncatum, previously reported in galleries of the ambrosia beetle Megaplatypus mutatus (Ceriani-Nakamurakare et al. 2016), was found to be sister to the clade containing G. kuroshium and G.euwallaceae. G. penicilliodes, G. fabiforme, G. fimbriisporum, G. pseudormiticum, and $G$. laricis were are all sister to the clade containing Graphium sp. (KSHB) and G. euwallaceae.

Fusarium kuroshium F. Na, J. D. Carrillo \& A. Eskalen, sp. nov. (Figures 1, 3, 5, and 8). MycoBank MB 821907.

Typification. United States, California. El Cajon, San Diego County. Surface of Euwallacea sp. galleries in infested California sycamore (Platanus racemosa), 14 December 2013, A. Eskalen (holotype BPI910340 = UCR3641).

Etymology. Derived from the common name of the ambrosia beetle vector, Kuroshio Shot Hole Borer (Stouthamer et al. 2017)

No teleomorph observed. F. kuroshium grown on PDA in the dark has average radial mycelial growth rates of $3.5 \mathrm{~mm} /$ day at $20^{\circ} \mathrm{C}$ and $6.5 \mathrm{~mm} /$ day at $25^{\circ} \mathrm{C}$ (Fig. 5). Colonies are initially white (A1) to cream (4A2) in color, and change to grayish red (10D4) with white aerial mycelium in 14 days. Color darkens to grayish ruby (12E5) with age. Reverse pigmentation of colonies grown on PDA in the dark were reddish brown (9D7) and reverse pigmentation becomes reddish brown (9E7) with age. Sporodochial pigmentation starts as light orange (5A4), becoming bluish green (25B8) to grayish green (25D7) with age. Cultures grown under continuous light are initially white (A1) to cream (4A2) in color, and change to brownish orange (5C4). Reverse colony pigmentation is also brownish orange (5C4) with a bluish gray (23D3) ring. Sporodochial pigmentation under continuous light starts reddish yellow (4A6) later becoming grayish green (25E7). Cultures grown on SNA formed colonies with no pigmentation and produced abundant aerial mycelium. Microconidia and macroconidia are produced on long aerial conidiophores with thin walls that are monophialidic and occasionally branched in conidiophores bearing macroconidia. Chlamydospores form in large numbers within hyphae and were also found in mature macroconidia. They are globose to ellipsoidal and can be intercalary or terminal in chains or singular with mostly a pale color and smooth, but can become pigmented with a rough texture containing bluish to brownish hues with time, 3.5 to $10.5 \times 3.0$ to $9.0 \mu \mathrm{m}$ total range. No sclerotia are present. Septation within macroconidia occurs over time as the conidia mature; up to five septa per conidium were observed in 14day-old cultures. Aerial microconidia mostly oval, occasionally reniform or fusiform, with 0 to 1 ( -2$)$ septation, zero-septate on SNA: 4.0 to $10.5 \times 2.5$ to $4.0 \mu \mathrm{m}$ total range, $6.4 \pm 1.4 \times 3.1 \pm 0.3 \mu \mathrm{m}$ on average (ex type: 4.5 to $7.5 \times 3$ to $4.0 \mu \mathrm{m}$ total range, $6.3 \pm 0.9 \times 3.3 \pm$ $0.4 \mu \mathrm{m}$ on average); two-celled oval one-septate on SNA: 5.5 to $13.0 \times 3.5$ to $5.0 \mu \mathrm{m}$ total range, $11.4 \pm 1.3 \times 4.0 \pm 0.70 \mu \mathrm{m}$ (ex type: 6.0 to $12.5 \times 3.0$ to $4.5 \mu \mathrm{m}$ total range, $9.3 \pm 1.8 \times 3.7 \pm 0.5 \mu \mathrm{m}$ on average). Macroconidia are sparsely distributed along with microconidia in culture but are found in abundance within sporodochial structures where they are clustered on thin walled conidiophores that are monophialidic. Macroconidia shape is relatively wide with the dorsal side more curved than the ventral side, wider toward the basal cells, which are barely notched. The apical cell morphology is papillate and round, 3 to $4(-5)$ septate formed on PDA and SNA after 14 days. Three septate on SNA in the dark: 15.0 to $20.5 \times 3.0$ to $7.5 \mu \mathrm{m}$ total range, $18.1 \pm 1.0 \times 5.6 \pm 0.6 \mu \mathrm{m}$ on average (ex type: 16.0 to $20.0 \times$ 5.0 to $6.5 \mu \mathrm{m}$ total range, $18.40 \pm 0.8 \times 5.7 \pm 0.5 \mu \mathrm{m}$ on average), on SNA under NUV light: 17.0 to $28.5 \times 5.5$ to $8.0 \mu \mathrm{m}$ total range, $24.7 \pm$ $1.8 \times 6.6 \pm 0.5 \mu \mathrm{m}$ on average (ex type: 20.0 to $27.0 \times 5.5$ to $8.0 \mu \mathrm{m}$ total range, $24.9 \pm 1.8 \times 6.6 \pm 0.7 \mu \mathrm{m}$ on average), on PDA in the dark: 16.0 to $20.5 \times 4.0$ to $6.5 \mu \mathrm{m}$ total range, $17.9 \pm 0.9 \times 5.3 \pm$ $0.5 \mu \mathrm{m}$ on average (ex type: 16.5 to $19.5 \times 4.5$ to $6.0 \mu \mathrm{m}$ total range, $17.7 \pm 0.8 \times 5.4 \pm 0.5 \mu \mathrm{m}$ on average); four septate on SNA in the dark: 17.5 to $24.0 \times 4.5$ to $7.5 \mu \mathrm{m}$ total range, $20.0 \pm 1.2 \times 5.8 \pm$ $0.5 \mu \mathrm{m}$ on average (ex type: 18.5 to $22.5 \times 5.0$ to $7.0 \mu \mathrm{m}$ total range, $20.2 \pm 1.2 \times 6.1 \pm 0.5 \mu \mathrm{m}$ on average), on SNA under NUV light: 22.5 to $32.0 \times 5.0$ to $9.0 \mu \mathrm{m}$ total range, $27.6 \pm 2.0 \times 6.8 \pm$ $0.7 \mu \mathrm{m}$ on average (ex type: 23.5 to $29.5 \times 6.0$ to $8.0 \mu \mathrm{m}$ total range, $26.5 \pm 1.5 \times 6.6 \pm 0.5 \mu \mathrm{m}$ on average), on PDA in the dark: 17.0 to $24.0 \times 4.5$ to $7.0 \mu \mathrm{m}$ total range, $19.4 \pm 1.3 \times 5.6 \pm 0.6 \mu \mathrm{m}$ on average (ex type: 18.0 to $21.0 \times 4.5$ to $6.5 \mu \mathrm{m}$ total range, $19.3 \pm 0.9 \times 5.5 \pm$ $0.6 \mu \mathrm{m}$ on average).

Known host range. Acer negundo, Albizia julibrissin, Baccharis salicifolia, Baccharis pilularis, Dombeya cacuminum, Erythrina humeana, Persea americana, Populus fremontii, Populus nigra, Platanus racemosa, Quercus agrifolia, Quercus suber, Ricinus

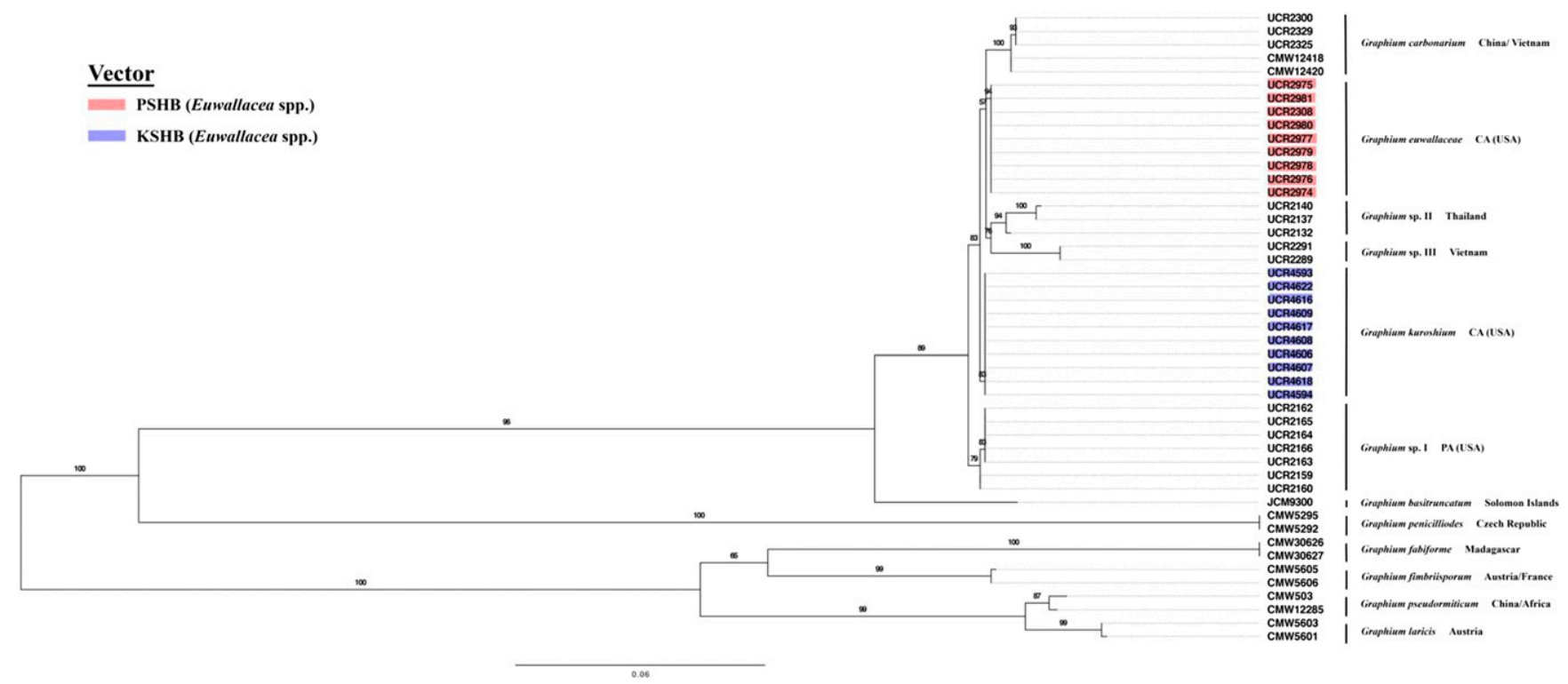

Fig. 2. Multilocus phylogenetic analysis of Graphium spp. conducted with two genes: ribosomal internal transcribed spacer (ITS) and elongation factor 1- $\alpha$ (EF1- $\alpha$ ). Diagram was constructed using IQ-TREE maximum likelihood method bootstrapped with 1,000 replications. The highlighted boxes indicate the beetle vector the fungi were recovered from. 
communis, Robinia pseudoacacia, Salix gooddingii, Salix laevigata, Salix lasiolepis, Tamarix ramosissima.

Ex-type culture. CBS142642 = Francis Na UCR3641

Fungal distribution. San Diego County, Orange County, California, U.S.A.

Additional specimens examined. United States, California. El Cajon, San Diego County. Surface of Euwallacea sp. galleries in P. racemosa, 2014, F. Na, UCR3644. United States, California. Fallbrook, San Diego County. Head of Euwallacea sp. in infested Persea americana, 2015, F. Na, UCR3651. United States, California. Bonsall, San Diego County. Surface of Euwallacea sp. galleries in $P$. americana, 2015, F. Na, UCR3653. United States, California. Bonsall, San Diego County. Surface of Euwallacea sp. galleries in P. americana, 2015, F. Na, UCR3654. United States, California. Bonsall, San Diego County. Head of Euwallacea sp. in infested $P$. americana, 2015, F. Na, UCR3653. United States, California. Bonsall, San Diego County. Head of Euwallacea sp. in infested P. americana, 2015, F. Na, UCR3657. United States, California. Bonsall, San Diego County. Head of Euwallacea sp. in infested P. americana, 2015, F. Na, UCR3659. United States, California. Bonsall, San Diego County. Head of Euwallacea sp. in infested P. americana, 2015, F. Na, UCR3660. United States, California. Escondido, San Diego County. Surface of Euwallacea sp. galleries

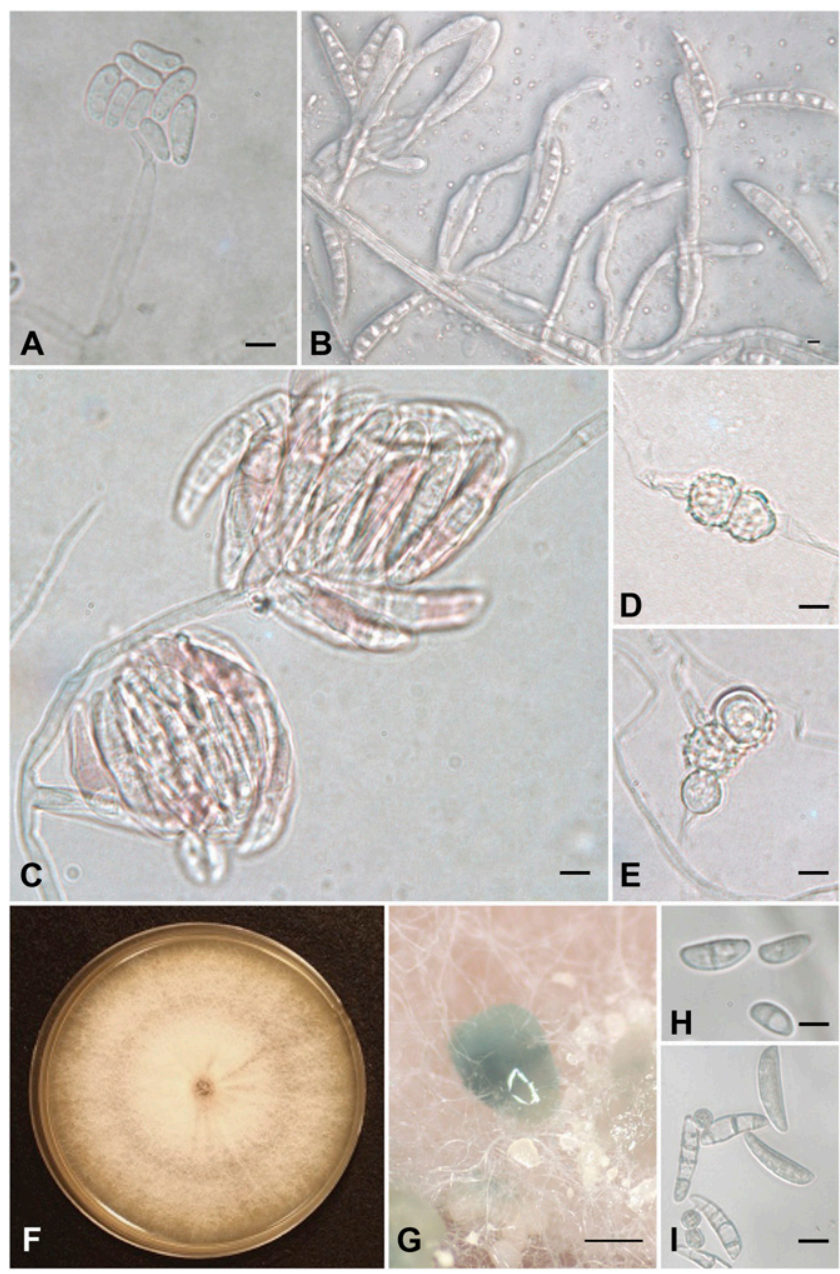

Fig. 3. A, B, D, I, Morphology of 14-day-old $F$. kuroshium cultures grown on synthetic low-nutrient agar (SNA) and C, E, H, F, G, on potato dextrose agar (PDA). A, Aseptate microconidium. B, Sporodochial conidiogenous philades forming fusiform-clavate macroconidia. C, Sporodochial conidiophores exhibiting reddish pigmentation. D, Paired rough-walled chlamydospores. E, Terminal rough-walled chlamydospores. F, Colony morphology on PDA at 2 weeks. G, Blue-green conidial masses formed in culture on PDA after 1 month. $\mathbf{H}, 0-1$ septate microconidia. I, Multiseptate macroconidia with chlamydospore rough-walled formation. $\mathbf{A}$ to $\mathbf{E}, \mathbf{H}$ and I, $5 \mu \mathrm{m}$; G, $1 \mathrm{~mm}$. in P. americana, 2015, F. Na, UCR3661. United States, California. Escondido, San Diego County. Surface of Euwallacea sp. galleries in $P$. americana, 2015, F. Na, UCR3662.

Comments. F. kuroshium resembles the related ambrosia fusaria clade (O'Donnell et al. 2015) member $F$. euwallaceae in various morphological features. F. kuroshium has been observed to differ from $F$. euwallaceae in colony pigmentation, which is lighter in color on PDA. Phylogenetic analysis shows that $F$. kuroshium is genetically distinct from $F$. euwallaceae, and that it is more closely related to several species of ambrosial Fusarium species obtained from Euwallacea sp. in Taichung, Taiwan, compared with $F$. euwallaceae.

Graphium kuroshium F. Na, J. D. Carrillo \& A. Eskalen, sp. nov. (Figures 2, 4, 6, and 8). MycoBank MP 821908.

Typification. United States, California. Fallbrook, San Diego County. Surface of Euwallacea sp. galleries in infested avocado (Persea americana), 20 November 2015, A. Eskalen (holotype BPI910341).

Etymology. Derived from the common name of the vector ambrosia beetle, Kuroshio Shot Hole Borer (Stouthamer et al. 2017)

G. kuroshium grown on PDA in the dark has radial mycelial growth rates on average of $1.3 \mathrm{~mm} /$ day at $20^{\circ} \mathrm{C}$ and $3.1 \mathrm{~mm} /$ day at $25^{\circ} \mathrm{C}$ (Fig. 6). Colony growth is initially white (1A), and colony changes in color to olive (3E4-3F3) with yellow green white margins (3B2) in 14-day-old cultures. Reverse pigmentation was olive (3D4) to a darker olive (3D4) in older cultures. Aerial mycelium on PDA was septate and hyaline. Colonies on OMA in the dark showed similar coloration to PDA with sparse mycelial growth with a rough surface. Colonies on PDA under continuous light are initially white (1A) and become olivaceous gray (3B2) with slate gray edges (3B2). Reverse pigmentation is stone gray (3E2). Colonies on OMA under continuous light are initially white (1A) and become olive (3E3) with

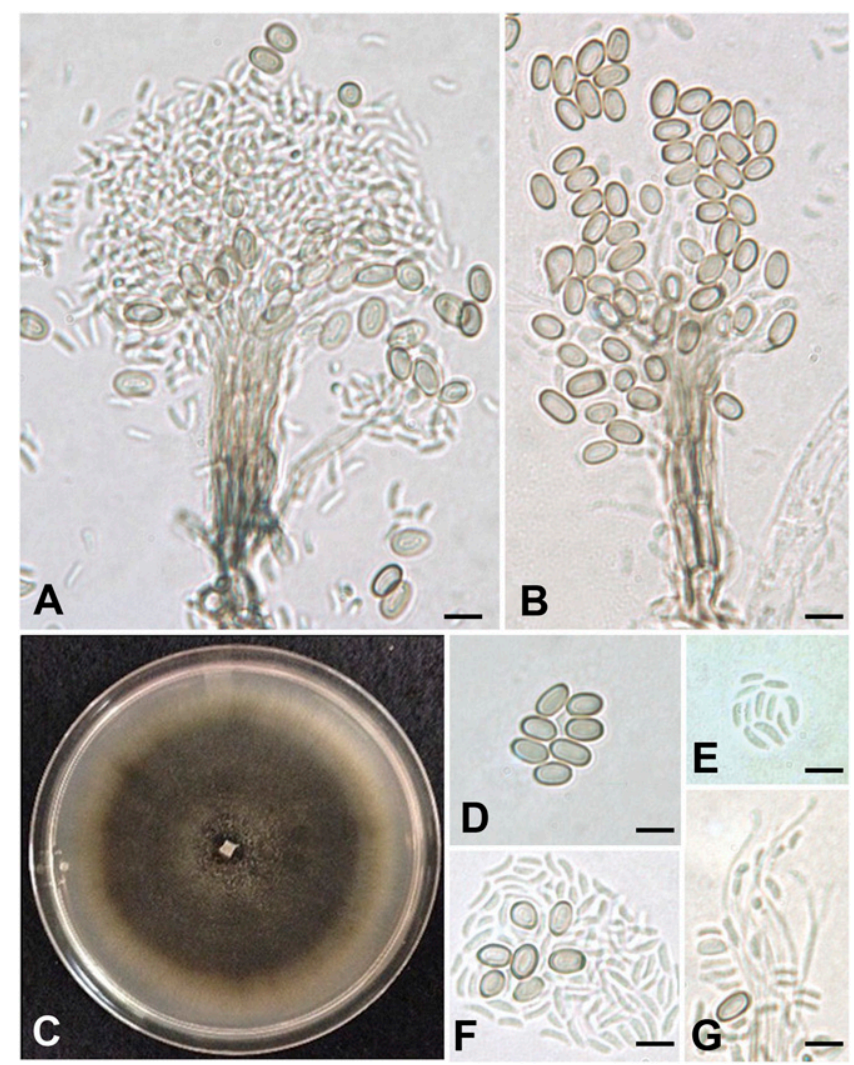

Fig. 4. A to G, Morphology of 14-day-old Graphium kuroshium cultures grown on potato dextrose agar (PDA). A, Synnema bearing cylindrical conidia with truncated ends. B, Synnema bearing ovoid conidia with thick walls. C, Colony morphology on PDA at 1 month. D, Ovoid conidia with thick walls. E, Cylindrical conidia with truncated ends. F, Both morphologically distinct conidia types. G, Conidiogenous philades forming on synnema. A, B, D to G, $5 \mu \mathrm{m}$. 
light edges (1A). Reverse pigmentation is olive (3D3). Synnemata consist of packed parallel hyphae $1.36 \pm 0.28 \mu \mathrm{m}$ in width. Synnemata structures are $9.88 \pm 2.24 \mu \mathrm{m}$ wide at the top, $6.12 \pm 1.98 \mu \mathrm{m}$ wide at the base of the fruiting body. Abundant hyphae rhizoid in shape present at the base of synnemata. Two types of aseptate conidia are observed from synnemata structures; hyaline cylindrical conidia with truncated ends on PDA in the dark: 3.0 to $7.2 \times 0.8$ to $2.3 \mu \mathrm{m}$ total range, $4.8 \pm 0.8 \times 1.4 \pm 0.3 \mu \mathrm{m}$ on average (ex type: 3.4 to $6.8 \times$ 1.0 to $1.9 \mu \mathrm{m}$ total range, $4.8 \pm 0.8 \times 1.4 \pm 0.2 \mu \mathrm{m}$ on average) and ovoid, thick walled, conidia on PDA in the dark: 3.4 to $5.6 \times 1.8$ to $3.6 \mu \mathrm{m}$ total range $4.6 \pm 0.4 \times 2.8 \pm 0.3 \mu \mathrm{m}$ on average (ex type: 3.7 to $5.6 \times 2.3$ to $3.1 \mu \mathrm{m}$ total range, $4.7 \pm 0.5 \times 2.8 \pm 0.2 \mu \mathrm{m}$ on average).

Known host range. Acer negundo, Albizia julibrissin, Baccharis salicifolia, Baccharis pilularis, Dombeya cacuminum, Erythrina humeana, Persea americana, Populus fremontii, Populus nigra, Platanus racemosa, Quercus agrifolia, Quercus suber, Ricinus communis, Robinia pseudoacacia, Salix gooddingii, Salix laevigata, Salix lasiolepis, Tamarix ramosissima.

Ex-type culture. CBS142643 = Francis Na UCR4593

Fungal distribution. San Diego County, California, U.S.A.

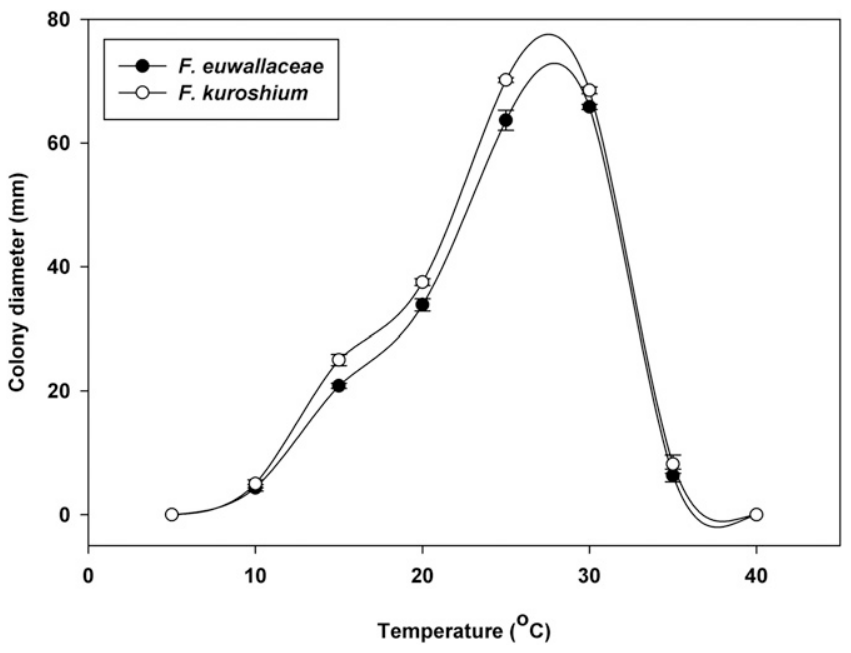

Fig. 5. Radial growth of Fusarium kuroshium and F. euwallaceae. Colony diameters of the resulting colonies were measured after 9 days of incubation. Vertical bars represent standard errors of the mean.

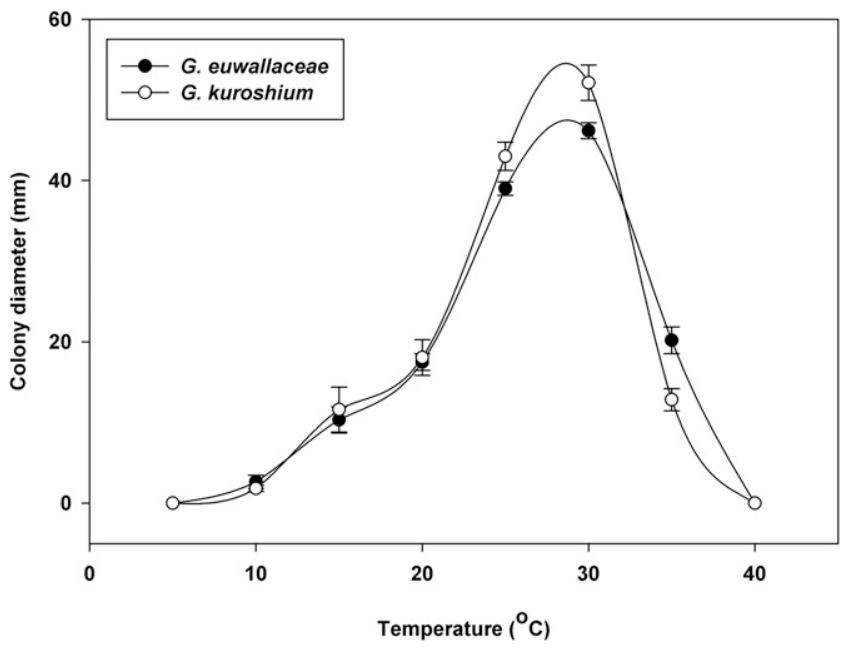

Fig. 6. Radial growth of Graphium kuroshium and G. euwallaceae. Colony diameters of the resulting colonies were measured after 14 days of incubation. Vertical bars represent standard errors of the mean.
Additional specimens examined. United States, California. Fallbrook, San Diego County. Surface of Euwallacea sp. galleries in P. americana, 2015, F. Na, UCR4594. United States, California. Bonsall, San Diego County. Surface of Euwallacea sp. galleries in P. americana, 2015, F. Na, UCR4606. United States, California. Bonsall, San Diego County. Surface of Euwallacea sp. galleries in P. americana, 2015, F. Na, UCR4607. United States, California. Bonsall, San Diego County. Head of Euwallacea sp. in infested P. americana, 2015, F. Na, UCR4608. United States, California. Bonsall, San Diego County. Head of Euwallacea sp. in infested P. americana, 2015, F. Na, UCR4609. United States, California. Escondido, San Diego County. Surface of Euwallacea sp. galleries in P. americana, 2015, F. Na, UCR4616. United States, California. Escondido, San Diego County. Surface of Euwallacea sp. galleries in P. americana, 2015, F. Na, UCR4617. United States, California. Escondido, San Diego County. Head of Euwallacea sp. in infested P. americana, 2015, F. Na, UCR4618. United States, California. Escondido, San Diego County. Head of Euwallacea sp. in infested P. americana, 2015, F. Na, UCR4622.

Comments. G. kuroshium colonies exhibit some morphological overlap to G. euwallaceae, an ambrosial Graphium species associated with polyphagous shot hole borer (Euwallacea sp. \#1) (O'Donnell et al. 2015), in culture. Conidial dimensions of ovoid conidia produced by G. kuroshium slightly differ from those produced by G. euwallaceae, as the average length of ovoid conidia produced by G. kuroshium was lower than the minimum length observed in G. euwallaceae (Lynch et al. 2016). Hyaline cylindrical conidia with truncated ends dimensions were within the range of observed dimensions in G. euwallaceae. Phylogenetic analysis shows that G. kuroshium is closely related to, but genetically distinct from $G$. euwallaceae.

Fungal recovery from KSHB female heads. F. kuroshium was the most abundant fungal species from macerated heads of KSHB recovered from avocado. F. kuroshium colony forming units (CFUs) were found to be present at the highest relative abundance $(83.5 \%)$ compared with $G$. kuroshium CFUs, which were present at a significantly lower abundance (16.5\%) (Fig. 7). Seventy percent of the beetles examined contained both $F$. kuroshium and G. kuroshium colonies with the remaining $30 \%$ containing exclusively $F$. kuroshium colonies. From the sampled beetles, G. kuroshium was not found exclusively in any mycangia and no beetle was found to contain Paracremonium pembeum, which Lynch et al. (2016) reported to

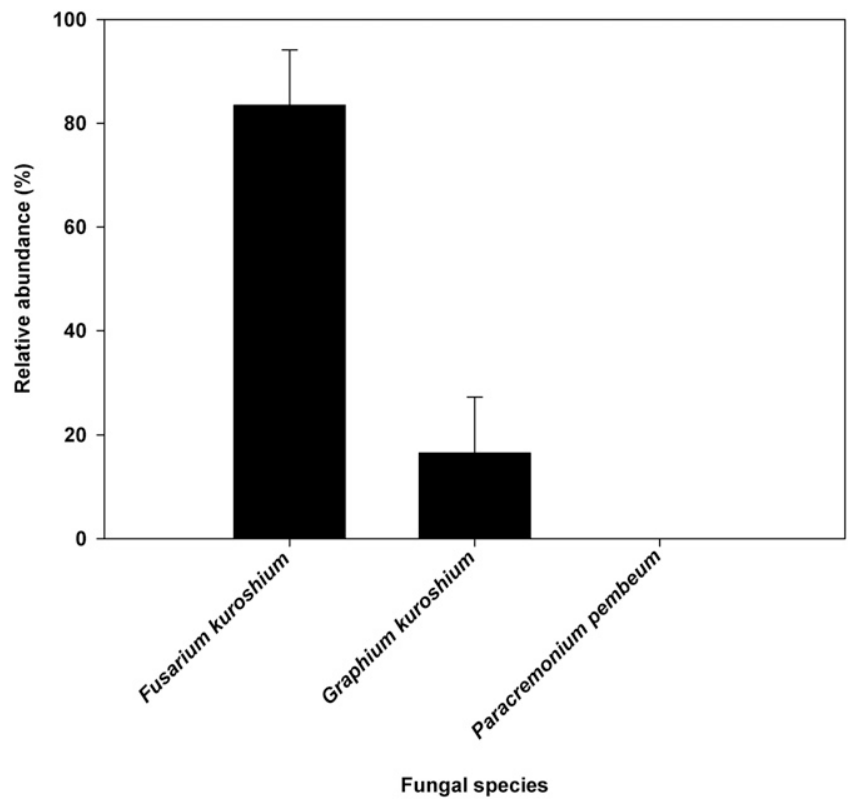

Fig. 7. Relative abundance of colonies Fusarium kuroshium, Graphium kuroshium, and Paracremonium pembeum recovered from KSHB female heads collected from avocado in San Diego County. Vertical lines represent standard error of the mean. 
be a facultative inhabitant in the mycangia of PSHB. No inhibition between mutualists was observed on the spread plates.

Pathogenicity assay. Avocado (cv. Zutano) seedlings infected with F. euwallaceae, F. kuroshium, G. euwallaceae, or G. kuroshium did not show dieback symptoms during the length of the experiment; however, sugar exudate resulting from mechanical damage of the phloem caused by the inoculation procedure was observed in most avocado seedlings used in the experiment including control treatments. Mean lesion lengths (Fig. 8) caused by F. kuroshium were found to be significantly smaller from those caused by $F$. euwallaceae $(P<0.001)$. Moreover, both Fusarium spp. tested were found to produce lesions significantly greater compared with the control $(P<0.01)$. Avocado seedlings inoculated with $F$. kuroshium produced lesions with an average length of $3.15 \pm 1.8 \mathrm{~cm}$, and seedlings inoculated with $F$. euwallaceae produced lesions with an average length of $6.89 \pm 2.1 \mathrm{~cm}$. Mean lesion lengths in plants infected with G. kuroshium (Fig. 8) did not significantly differ from those in plants infected with $G$. euwallaceae $(P>0.05)$ but both were significantly greater than the control $(P<0.001)$. Plants infected with G. euwallaceae showed lesions with an average length of $6.4 \pm 2.8 \mathrm{~cm}$, and those infected with $G$. kuroshium showed lesions with an average length of $7.6 \pm 4.1 \mathrm{~cm}$. There were significant differences in lesion length $(P<0.001)$ from $F$. kuroshium compared with all other fungi inoculated, but it was still significantly greater than the control $(P<$ 0.05). Control seedlings treated with sterile agar plugs produced a wound response with a mean length of $0.6 \pm 0.1 \mathrm{~cm}$. Fungal recovery for all species ranged from 90 to $100 \%$ and none of the inoculated fungi were recovered from the controls.

\section{Discussion}

Multigene molecular analyses of both $F$. kuroshium (Fig. 1) and G. kuroshium (Fig. 2) associated with KSHB reveal a distinct phylogeny compared with closely related AFC fusaria and Graphium species, respectively. PSHB and KSHB invading California are morphologically indistinguishable members of the Euwallacea genus, but have been previously found to be genetically distinct at the COI locus as well as other nuclear genes tested (O'Donnell et al. 2015; Stouthamer et al. 2017) and exclusively associated with distinct Fusarium spp. as well as Graphium spp. based on results from the current study. Although there is morphological overlap in AFC fusaria (Freeman et al. 2013; Kasson et al. 2013; O'Donnell et al. 2015) as well as in Graphium spp. (Lynch et al. 2016) associated with invasive SHB,

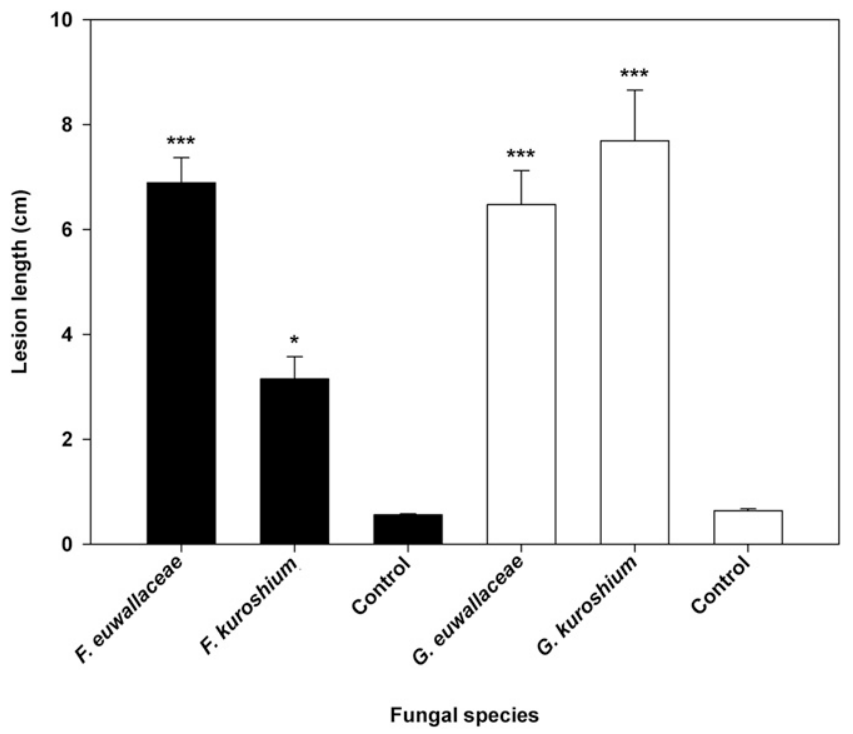

Fig. 8. Mean lesion length of xylem discoloration caused by Fusarium euwallaceae, F. kuroshium, Graphium euwallaceae, and G. kuroshium in 2-year-old avocado seedlings. Vertical lines represent standard error of the mean. $P$-values are represented as ${ }^{* *}(P<0.001),{ }^{* *}(P<0.001)$, and ${ }^{*}(P<0.05)$ according to a Tukey's bootstrap test with confidence level $\alpha=0.05$. molecular-based identification at informative loci such as TEF1- $\alpha$, RPB-1, and RPB-2 is the best approach for proper identification of PSHB and KSHB symbionts. A PCR-based assay has already been described that can distinguish AFC fusaria (Short et al. 2017) and may be useful for diagnostic identification of these Fusarium spp. symbionts with sympatric geography. Based on morphological and molecular differences of these two fungal symbionts of KSHB, we propose the names $F$. kuroshium sp. nov. and G. kuroshium sp. nov. based on the exclusive association with the insect vector KSHB (Euwallacea sp. nr. fornicatus) in California. Both populations of fungal symbionts associated with PSHB and KSHB invasive to California are shown here to be pathogenic on avocado and were first observed in Los Angeles and San Diego counties, respectively, causing FD symptoms on 15 different host species (http://eskalenlab.ucr.edu/ shotholeborerhosts.html). The two pest disease complexes KSHBFD and PSHB-FD should be considered distinct, but collective threats spreading and adversely impacting woody hosts throughout California.

Ambrosia beetles from the Euwallacea genus have invaded the U.S. on multiple occasions (CABI 2015; Hulcr and Dunn 2011; Hulcr and Stelinski 2017; Kasson et al. 2013; O'Donnell et al. 2015; Stouthamer et al. 2017), carrying fungal symbionts that have evolved with them in mutualisms that provide the beetles with nutritional supplement from low-nutrient xylem tissue, while the fungi receive reliable dispersion and direct inoculation into plant hosts (Beaver 1989). The exclusive relationships are maintained upon invasion, which is seen in PSHB-FD as it has invaded California (Eskalen et al. 2013) and Israel (Mendel et al. 2012), vectoring the same previously described symbionts $(F$. euwallaceae; Freeman et al. 2013; G. euwallaceae, and P. pembeum; Lynch et al. 2016) in all locales. The present study suggests an exclusive relationship is also present in the KSHB-FD invasion of California with fungal mutualists $F$. kuroshium and G. kuroshium classifying KSHB-FD as a unique pest-disease complex. These invasive pest-disease complexes may also be similarly unique for other areas of the U.S. and other countries affected by invasive Euwallacea spp., but it is currently unknown to what extent these populations of invasive Euwallacea spp. can obtain new symbionts in what have been previously described as "host shifts" in which Euwallacea spp. AFC mutualists can change associations with the insect hosts over time (O'Donnell et al. 2015). In addition to AFC mutualists, other mutualists such as Graphium spp. may also undergo host shifts in areas where more than one population of Euwallacea spp. exists. PSHB and KSHB are currently both present in Orange County and parts of Los Angeles County (http://eskalenlab. ucr.edu/distribution.html) and present a situation in which potential "host shifts" may occur and should be investigated to find if new combinations of Fusarium spp. and Graphium spp. are possible and what effects they may have on severity of FD in plant hosts.

Fusarium and Graphium species from other Euwallacea spp. in southeast Asia included in this study allowed for investigation into the potential origin of the symbionts recovered from KSHB in California. F. kuroshium obtained from KSHB was shown to be closely related to some AFC isolates recovered from Euwallacea sp. in Taiwan (Fig. 1), yet distant from F. euwallaceae associated with PSHB from California and Israel. The placement of two AFC isolates (Fusarium sp. AF-13 Taiwan) originating from Taiwan with the closest relation to $F$. kuroshium suggests a potential origin of KSHB-FD based on phylogenetic relationship, corroborating previous findings from Stouthamer et al. (2017) where analysis of the cytochrome oxidase $c$ subunit I (COI) mitochondrial gene in Euwallacea spp. confirmed KSHB haplotype (H22) found in California matched one of the four KSHB haplotypes found in Taiwan. Fusarium spp. AF-14 also show close relation to $F$. kuroshium and $F$. euwallaceae as they are resolved in AFC clade B (Kasson et al. 2013; O'Donnell et al. 2015) but form a distinct phylogenetic lineage along with Fusarium sp. AF-15 Taiwan, which is closely related to Fusarium sp. AF-4 from Pennsylvania, U.S.A. Fusarium sp. AF-16 Taiwan is notable as a new AFC species that forms a unique lineage distinct from previously described AFC species (Kasson et al. 2013; O'Donnell et al. 2015). The diversity present in Taiwan isolates included in this study 
may be explained by greater diversity of host Euwallacea spp. in this geographic region and/or the coevolution as exclusive fungal mutualists of the vectors. Alternatively, AFC may associate with multiple Euwallacea spp. in Taiwan, creating opportunity for parasexual or potential sexual fungal interactions with other AFC fusaria present in the native habitat, increasing diversity. Further genomic sequencing and evolutionary analyses of populations of Taiwanese strains will allow for further testing of the relative contributions of these mechanisms to the observed genetic diversity.

The other common fungal symbiont of KSHB, G. kuroshium, was also shown to be genetically distinct from G. euwallaceae associated with PSHB using a two-gene phylogeny (Fig. 2, ITS, TEF-1 $\alpha$ ). Although we were unable to completely resolve the relationship of G. kuroshium to other Graphium spp. associated with Euwallacea spp. in Taiwan in the current study, as was possible for the AFC Fusarium spp., there is opportunity to elucidate phylogeographic patterns from this group in future studies by sampling from more native southeast Asian areas. Understanding the geographical origins of the ancestors of the fungi associated with invasive Euwallacea spp. may reveal relevant locations to narrow the search for natural control methods using natural predators, parasites, and other biotic/abiotic interactions that adversely affect these pests and can potentially provide an effective management strategy of these invasive pests.

In this study, the previously described fungal species, $P$. pembeum (Lynch et al. 2016), was not recovered from KSHB female heads and/or in wood tissue from galleries. This finding was notable because $P$. pembeum has previously been recovered with $F$. euwallaceae and $G$. euwallaceae from both the heads of the PSHB female beetles and their galleries (Lynch et al. 2016). The role of P. pembeum in the life cycle is unclear, but has been previously reported to be recovered at higher abundance from beetle larvae in castor bean and avocado than other hosts examined (Freeman et al. 2016). In this study, we only cultured fungi from adult females and gallery walls; we did not attempt to recover fungi from preadult stages of the life cycle, which may explain why we were not able to recover Paracremonium spp., but it should be investigated further to see if Paracemonium spp. are mutualists of KSHB.

F. kuroshium and G. kuroshium associated with the KSHB-FD complex were found to be pathogens on healthy young avocado plants and their status as a causal agent was confirmed by Koch's postulates performed in this study (Fig. 8). The symptom severity of F. kuroshium observed during the pathogenicity trial was significantly less $(P<$ 0.05 ) when compared with $F$. euwallaceae, with the mean lesion size $46 \%$ of the size to that of $F$. euwallaceae over a 4-week time frame. Conversely, G. kuroshium was found to cause lesion sizes comparable to those of $G$. euwallaceae. The mode of ambrosia beetle damage in susceptible hosts from PSHB-FD and KSHB-FD is likely "Mode 2" suggested by Hulcr and Stelinski (2017), where mass accumulation of the pests on stressed trees leads to significant damage. Although both symbionts of KSHB are shown here as relatively weak pathogens, it is likely a combination of attacks by the beetle and inoculations of their mutualists that lead to FD symptoms, which can increase in severity when mass attacks accumulate on a susceptible host. In San Diego County, KSHB-FD has already been reported in commercial avocado groves and is causing branch dieback in multiple locations (S. Lynch, personal communication), but it is currently unknown what impact the pests will have on avocado production. Therefore, the pests should be monitored in these areas to gain a better understanding of the extent of damage caused in California. More plant species in urban forests and native vegetation than the agriculturally important commodity avocado are affected by this disease, as the reproductive host range of PSHB-FD has been previously described (49 hosts) (Eskalen et al. 2013; Mendel et al. 2012), but the host range of KSHB-FD is not yet fully resolved. More investigation into the full reproductive host range and the biotic and abiotic factors that contribute to host susceptibility and defense responses to PSHB-FD/KSHB-FD can aid in elucidating why these invasive pest-disease complexes are destructive in invaded areas as well as their native habitat.

The classification of KSHB-FD as a unique complex invading California distinguishes this new complex from the closely related
PSHB-FD complex. There is more diversity among the fungal symbionts of ambrosia beetles previously reported in their native habitat (Beaver and Liu 2010; Hulcr and Cognato 2010; Li et al. 2015; Stouthamer et al. 2017). These invasive beetles present in California and other invaded areas are likely subsamples of a larger diverse Asian population. This may explain the apparent exclusive relationships that are found among fungal symbionts recovered from ambrosia beetles from the E. fornicatus complex in invaded areas. The extent of these exclusive symbiotic relationships in their native habitats is currently unknown and it may be possible that the beetles are associated with multiple species of AFC fusaria, Graphium spp., Paracremonium spp., and/or other unidentified mutualists. Since Euwallacea spp. are completely dependent on their mutualists to provide nutrition from nutrient-poor xylem tissue (Baker and Norris 1968; Beaver 1989), it would be informative to explore the pathogenicity and virulence of fungal symbionts associated with Euwallacea spp. in their native regions on susceptible hosts to investigate if there is any correlation to beetle fecundity. Although $95 \%$ of ambrosia beetle-fungal complexes are reported to be economically harmless (Hulcr and Stelinski 2017), biological information from these complexes, including species descriptions of both the insect and pathogen(s) involved in native and invasive SHB-fungi complexes, both destructive and nondestructive, would be highly informative. This information should extend past scientific literature and should be recognized by government agencies worldwide to regulate and restrict the movement of new, potentially destructive, SHB-fungi complexes into other uninfested areas outside of their native habitat.

\section{Acknowledgments}

We wish to thank D. H. Wang and K. Y. Sugino for assistance with field collection and greenhouse setup. Phylogenetic analyses were performed on high performance computing (HPCC) resources on the UC Riverside Institute for Integrative Genome Biology supported by funding for hardware equipment purchases under grants from the National Science Foundation. The funders had no role in study design, data collection and analysis, decision to publish, or preparation of the manuscript.

\section{Literature Cited}

Alonso-Zarazaga, M. A., and Lyal, C. H. 2009. A catalogue of family and genus group names in Scolytinae and Platypodinae with nomenclatural remarks (Coleoptera: Curculionidae). Zootaxa 2258:112-127.

Aoki, T., O'Donnell, K., and Scandiani, M. M. 2005. Sudden death syndrome of soybean in South America is caused by four species of Fusarium: Fusarium brasiliense sp. nov., F. cuneirostrum sp. nov., F. tucumaniae, and F. virguliforme. Mycoscience 46:162-183.

Baker, J. M., and Norris, D. M. 1968. A complex of fungi mutualistically involved in the nutrition of the ambrosia beetle Xyleborus ferrugineus. J. Invertebr. Pathol. 11:246-250.

Bates, D., Mächler, M., Bolker, B., and Walker, S. 2015. Fitting linear mixedeffects models using lme4. J. Stat. Soft. 67:1-48.

Beaver, R. A. 1989. Insect-fungus relationships in the bark and ambrosia beetles. Pages 121-143 in: Insect-fungus Interactions. N. Wilding, N. M. Collins, P. M. Hammond, and J. F. Webber, eds. Academic Press, London.

Beaver, R. A., and Liu, L. Y. 2010. An annotated synopsis of Taiwanese bark and ambrosia beetles, with new synonymy, new combinations and new records (Coleoptera: Curculionidae: Scolytinae). Zootaxa 2602:1-47.

Beaver, R. A., Sittichaya, W., and Liu, L. Y. 2014. A synopsis of the scolytine ambrosia beetles of Thailand (Coleoptera: Curculionidae: Scolytinae). Zootaxa 3875:1-82.

CABI. 2015. Euwallacea fornicatus Distribution Map. https://www.cabi.org/isc/ datasheet/57163.

Cenis, J. L. 1992. Rapid extraction of fungal DNA for PCR amplification. Nucleic Acids Res. 20:2380.

Ceriani-Nakamurakare, E., Slodowicz, M., Gonzalez-Audino, P., Dolinko, A., and Carmarán, C. 2016. Mycobiota associated with the ambrosia beetle Megaplatypus mutatus: threat to poplar plantations. Forestry 89:191-200.

Cognato, A. I., Hoebeke, E. R., Kajimura, H., and Smith, S. M. 2015. History of the exotic ambrosia beetles Euwallacea interjectus and Euwallacea validus (Coleoptera: Curculionidae: Xyleborini) in the United States. J. Econ. Entomol. 108:1129-1135.

Cooperband, M. F., Stouthamer, R., Carrillo, D., Eskalen, A., Thibault, T., Cossé, A. A., Castrillo, L. A., Vandenberg, J. D., and Rugman-Jones, P. F. 2016. Biology of two members of the Euwallacea fornicatus species complex (Coleoptera: Curculionidae: Scolytinae), recently invasive in the U.S.A., reared on an ambrosia beetle artificial diet. Agr. For. Entomol. 18:223-237.

Danthanarayana, W. 1968. The distribution and host-range of the shot-hole borer (Xyleborus fornicatus Eich.) of tea. Tea Q. 39:61-69. 
Eskalen, A., Gonzalez, A., Wang, D. H., Twizeyimana, M., and Mayorquin, J. S. 2012. First report of a Fusarium sp. and its vector tea shot hole borer (Euwallacea fornicatus) causing Fusarium dieback on avocado in California. Plant Dis. 96:1070.

Eskalen, A., Stouthamer, R., Lynch, S. C., Rugman-Jones, P. F., Twizeyimana, M., Gonzalez, A., and Thibault, T. 2013. Host range of Fusarium dieback and its ambrosia beetle (Coleoptera: Scolytinae) vector in southern California. Plant Dis. 97:938-951

Fox, J., Weisberg, S., Adler, D., Bates, D., Baud-Bovy, G., Ellison, S., Firth, D., Friendly, M., Gorjanc, G., Graves, S. and Heiberger, R. 2016. Package 'CAR'.

Freeman, S., Sharon, M., Dori-Bachash, M., Maymon, M., Belausov, E., Maoz, Y., Margalit, O., Protasov, A., and Mendel, Z. 2016. Symbiotic association of three fungal species throughout the life cycle of the ambrosia beetle Euwallacea nr. fornicatus. Symbiosis 68:115-128.

Freeman, S., Sharon, M., Maymon, M., Mendel, Z., Protasov, A., Aoki, T., Eskalen, A., and O'Donnell, K. 2013. Fusarium euwallaceae sp. nov.-a symbiotic fungus of Euwallacea sp., an invasive ambrosia beetle in Israel and California. Mycologia 105:1595-1606.

Gams, W., Hoekstra, E. S., and Aproot, A. 1998. CBS Course of Mycology. 4th ed. Centraalbureau voor Schimmelcultures, Baarn, The Netherlands.

Hulcr, J., and Cognato, A. I. 2010. Repeated evolution of crop theft in fungusfarming ambrosia beetles. Evolution 64:3205-3212.

Hulcr, J., and Dunn, R. R. 2011. The sudden emergence of pathogenicity in insectfungus symbioses threatens native forest ecosystems. Proc. Royal Soc. B 278: 2866-2873.

Hulcr, J., Rountree, N. R., Diamond, S. E., Stelinski, L. L., Fierer, N., and Dunn, R. R. 2012. Mycangia of ambrosia beetles host communities of bacteria. Microb. Ecol. 64:784-793

Hulcr, J., and Stelinski, L. L. 2017. The ambrosia symbiosis: from evolutionary ecology to practical management. Annu. Rev. Entomol. 62:285-303.

Jacobs, K., Bergdahl, D. R., Wingfield, M. J., Halik, S., Seifert, K. A., Bright, D. E., and Wingfield, B. D. 2004. Leptographium wingfieldii introduced into North America and found associated with exotic Tomicus piniperda and native bark beetles. Mycol. Res. 108:411-418

Kajimura, H., and Hijii, N. 1992. Dynamics of the fungal symbionts in the gallery system and the mycangia of the ambrosia beetle Xylosandrus mutilatus (Blandford) (Coleoptera: Scolytidae) in relation to its life history. Ecol. Res. 7:107-117.

Kasson, M. T., O’Donnell, K., Rooney, A. P., Sink, S., Ploetz, R. C., Ploetz, J. N., Konkol, J. L., Carrillo, D., Freeman, S., Mendel, Z., Smith, J. A., Black, A. W., Hulcr, J., Bateman, C., Stefkova, K., Campbell, P. R., Geering, A. D. W., Dann, E. K., Eskalen, A., Mohotti, K., Short, D. P. G., Aoki, T., Fenstermacher, K. A., Davis, D. D., and Geiser, D. M. 2013. An inordinate fondness for Fusarium: Phylogenetic diversity of fusaria cultivated by ambrosia beetles in the genus Euwallacea on avocado and other plant hosts. Fungal Genet. Biol. 56:147-157.

Kirisits, T. 2007. Fungal associates of European bark beetles with special emphasis on the ophiostomatoid fungi. Pages 181-236 in: Bark and Wood Boring Insects in Living Trees in Europe, a Synthesis. Springer, Dordrecht, Netherlands.

Lynch, S. C., Twizeyimana, M., Mayorquin, J. S., Wang, D. H., Na, F., Kayim, M., Kasson, M. T., Thu, P. Q., Bateman, C., Rugman-Jones, P., Hulcr, J., Stouthamer, R., and Eskalen, A. 2016. Identification, pathogenicity and abundance of Paracremonium pembeum sp. nov. and Graphium euwallaceae sp. nov.- - two newly discovered mycangial associates of the polyphagous shot hole borer (Euwallacea sp.) in California. Mycologia 108:313-329.

Li, Y., Simmons, D. R., Bateman, C. C., Short, D. P. G., Kasson, M. T., Rabaglia, R. J., and Hulcr, J. 2015. New fungus-insect symbiosis: culturing, molecular, and histological methods determine saprophytic polyporales mutualists of Ambrosiodmus ambrosia beetles. PLoS ONE 10:e137689.

Mendel, Z., Protasov, A., Sharon, M., Zveibil, A., Ben Yehuda, S., O’Donnell, K., Rabaglia, R., Wysoki, M., and Freeman, S. 2012. An Asian ambrosia beetle Euwallacea fornicatus and its novel symbiotic fungus Fusarium sp. pose a serious threat to the Israeli avocado industry. Phytoparasitica 40:235-238.

Munsell Color. 1976. Munsell book of color: matte finish collection. Munsell Color, Grand Rapids, MI.
Nguyen, L.-T., Schmidt, H. A., von Haeseler, A., and Minh, B. Q. 2015. IQ-TREE: a fast and effective stochastic algorithm for estimating maximum-likelihood phylogenies. Mol. Biol. Evol. 32:268-274.

Nirenberg, H. 1976. Untersuchungen uber die morphologische und biologische Differenzierung in der Fusarium-Sektion Liseola. Mitt. Biol. Bundesanst. LandForstw. 169:1-117.

OC Parks. 2017. Shot Hole Borer: Managing the Invasive Beetle. https://oc-parks-gis.maps. arcgis.com/apps/Cascade/index.html?appid=680fd0c9e73f4857a8477791f7ee796f.

O’Donnell, K., Cigelnik, E., and Nirenberg, H. I. 1998. Molecular systematics and phylogeography of the Gibberella fujikuroi species complex. Mycologia 90: 465-493.

O’Donnell, K., Sarver, B. A. J., Brandt, M., Chang, D. C., Noble-Wang, J., Park, B. J., Sutton, D. A., Benjamin, L., Lindsley, M., Padhye, A., Geiser, D. M., and Ward, T. J. 2007. Phylogenetic diversity and microsphere array-based genotyping of human pathogenic fusaria, including isolates from the multistate contact lensassociated U.S. keratitis outbreaks of 2005 and 2006. J. Clin. Microbiol. 45 2235-2248.

O’Donnell, K., Sink, S., Libeskind-Hadas, R., Hulcr, J., Kasson, M. T., Ploetz, R. C., Konkol, J. L., Ploetz, J. N., Carrillo, D., Campbell, A., Duncan, R. E., Liyanage, P. N., Eskalen, A., Na, F., Geiser, D. M., Bateman, C., Freeman, S., Mendel, Z., Sharon, M., Aoki, T., Crossé, A. A., and Rooney, A. P. 2015. Discordant phylogenies suggest repeated host shifts in the Fusarium-Euwallacea ambrosia beetle mutualism. Fungal Genet. Biol. 82:277-290.

O’Donnell, K., Sutton, D. A., Rinaldi, M. G., Sarver, B. A. J., Balajee, S. A., Schroers, H. J., Summerbell, R. C., Robert, V. A. R. G., Crous, P. W., Zhang, N., Aoki, T., Jung, K., Park, J., Lee, Y. H., Kang, S., Park, B., and Geiser, D. M. 2010. Internet-accessible DNA sequence database for identifying fusaria from human and animal infections. J. Clin. Microbiol. 48:3708-3718.

Ploetz, R. C., Hulcr, J., Wingfield, M. J., and de Beer, Z. W. 2013. Destructive tree diseases associated with ambrosia and bark beetles: black swan events in tree pathology? Plant Dis. 97:856-872.

Rabaglia, R. J., Dole, S. A., and Cognato, A. I. 2006. Review of American Xyleborina (Coleoptera: Curculionidae: Scolytinae) occurring north of Mexico, with an illustrated key. Ann. Entomol. Soc. Am. 99:1034-1056.

Short, D. P., O’Donnell, K., Stajich, J. E., Hulcr, J., Kijimoto, T., Berger, M. C., Macias, A. M., Spahr, E. J., Bateman, C. C., Eskalen, A., and Lynch, S. C. 2017. PCR multiplexes discriminate Fusarium symbionts of invasive Euwallacea ambrosia beetles that inflict damage on numerous tree species throughout the United States. Plant Dis. 101:233-240.

Smith, S. M., and Hulcr, J. 2015. Scolytus and other economically important bark and ambrosia beetles. Pages 495-531 in: Bark Beetles: Biology and Ecology of Native and Invasive Species. Elsevier, London.

Stamatakis, A. 2014. RAxML version 8: a tool for phylogenetic analysis and postanalysis of large phylogenies. Bioinformatics 30:1312-1313.

Stouthamer, R., Rugman-Jones, P., Thu, P. Q., Eskalen, A., Thibault, T., Hulcr, J., Wang, L. J., Jordal, B. H., Chen, C. Y., Cooperband, M., and Lin, C. S. 2017. Tracing the origin of a cryptic invader: phylogeography of the Euwallacea fornicatus (Coleoptera: Curculionidae: Scolytinae) species complex. Agric. For. Entomol. 19:366-375.

Thompson, J. D., Gibson, T. J., Plewniak, F., Jeanmougin, F., and Higgins, D. G. 1997. The CLUSTAL X windows interface: Flexible strategies for multiple sequence alignment aided by quality analysis tools. Nucleic Acids Res. 25: 4876-4882.

Twizeyimana, M., Forster, H., McDonald, V., Wang, D. H., Adaskaveg, J. E., and Eskalen, A. 2013. Identification and pathogenicity of fungal pathogens associated with stem-end rot of avocado in California. Plant Dis. 97:1580-1584.

White, T. J., Bruns, T., Lee, S., and Taylor, J. 1990. Amplification and direct sequencing of fungal ribosomal RNA genes for phylogenetics. Pages 315-322 in: PCR Protocols: A Guide to Methods and Applications. M. A. Innis, D. H. Gelfand, J. J. Sninsky, and T. J. White, eds. Academic Press, San Diego, CA.

Wickham, H. 2011. The split-apply-combine strategy for data analysis. J. Stat Soft. 40:1-29. 